\title{
Compressive properties of Min-mod-type limiters in modelling shockwave-containing flows
}

\author{
Guangning $\mathrm{Li}^{1,2}$. Dinesh Bhatia ${ }^{3}$ Jian Wang ${ }^{2}$
}

Received: 24 August 2019 / Accepted: 26 April 2020 / Published online: 12 May 2020

(c) The Author(s) 2020

\begin{abstract}
The long-ignored compressive properties of Min-mod-type limiter is investigated in this manuscript by demonstrating its potential in numerically modelling shockwave-containing flows, especially in shock wave/boundary layer interaction (SWBLI) problems. Theoretical studies were firstly performed based on Sweby's total variation diminishing (TVD) limiter region and Spekreijse's monotonicity-preserving limiter region to indicate Min-mod-type limiters' compressive properties. The influence of limiters on the solution accuracy was evaluated using a hybrid-order analysis method based on the gridindependent study in three typical shockwave-containing flows. The conclusions are that, Min-mod-type limiter can be utilized as a dissipative and/or compressive limiter, but depending on the reasonable value of the compression parameter. The compressive Min-mod limiter tends to be more attractive in modelling shockwave-containing flows as compared to other commonly preferred limiters because of its stable computational process and its high-resolution predictions. However, the compressive Min-mod limiter may suffer from its slightly poor convergence, as that observed in other commonly accepted smooth limiters in modelling SWBLI problems.
\end{abstract}

Keywords TVD $\cdot$ Min-mod limiter $\cdot$ SWBLI $\cdot$ Grid independent analysis $\cdot$ MUSCL approach

\section{Introduction}

The shockwave/boundary layer interactions (SWBLI) have been greatly attractive in engineering and scientific research for more than 50 years [1-5]. To investigate the SWBLI problems [6-8], the high-order (at least third-order) shockcapturing schemes [9-12] are the general choice. However, the high-order schemes are less robust, difficult to code and practice, especially in solving SWBLI problems which

Technical Editor: André Cavalieri.

Jian Wang

j.wang@kingston.ac.uk

1 Shaanxi Aerospace Flight Vehicle Design Key Laboratory, School of Astronautics, Northwestern Polytechnical University, Xi' an 710072, Shaanxi, China

2 School of Engineering and Environment, Faculty of Science, Engineering and Computing, Kingston University, Roehampton Vale Campus, Friars Avenue, London SW15 3DW, UK

3 School of Aerospace, Faculty of Science and Engineering, The University of Nottingham Ningbo China, Ningbo 315000, Zhejiang, China contain numerous steep gradients in the form of shockwaves and relatively thin thickness of boundary layers. For industrial applications, there are very few routinely utilized working CFD codes embedding higher than second-order accuracy. The classical second-order upwind schemes [13-16] are always preferable in practical aerodynamics because of their simplicity, efficiency, flexibility and robustness in practice.

The main approaches of conducting second-order schemes for strong shockwave-containing flows are MUSCL and non-MUSCL approach [17-20]. Although the nonMUSCL-based schemes have shown to provide excellent accuracy, they suffer from unsound convergence properties due to the inherent non-differentiable characteristics. As compared to the non-MUSCL-based schemes, the MUSCLbased approach is more prevalent in engineering due to its inborn simplicity and efficiency in modelling complex flows. To achieve the second-order accuracy, the MUSCL method [21] proposed by Van Leer is commonly adopted. The linear interpolation method used in designing a second-order scheme can cause spurious oscillations in steep gradient regions [22]. Therefore, the MUSCL-based linear interpolation needs to be improved in a special manner, i.e. the limiter 
functions, when the flow solution contains shockwave or other discontinuities. The application of limiter function in designing upwind schemes can suppress the numerical oscillations, achieve the monotonically converged solutions with the aimed second-order accuracy. In this paper, the authors mainly focus on the classical second-order schemes, with the emphasis being laid on the influence of limiter functions on the accuracy and the convergence behaviour in numerical simulations of SWBLI problems.

Existing literature shows that extensive research has already been conducted on developing accurate and robust limiters. The most significant breakthrough in this field is Sweby's second-order TVD limiter region [23] based on the one-dimensional scalar conservation law as shown in Fig. 1a. However, Goodman and Leveque [24] have stated that TVD schemes in two-dimensional cases can only achieve first-order accuracy at most. To achieve secondorder accuracy in multi-dimensional problems, Spekreijse [25] extended Sweby's TVD limiter region to monotonic limiter region as shown in Fig. 1b. Barth and Jespersen [26] derived a multi-dimensional limiter suitable for unstructured grids based on Spekreijse's monotonic limiter region. In the application of the limiter in the upwind scheme, the frequently encountered problem is that it may severely hamper the numerical solution convergence. This phenomenon is even more pronounced for a non-differentiable limiter. Venkatakrishnan [27] devised a limiter function, in which a threshold parameter based on local cell size was added. And then, the solutions can converge to the steady state as expected, and in regions where numerical oscillations were below the selected threshold, the limiter could be effectively switched off. This modification to limiter is similar to that of van Albada et al. [28] in a different context via the problem of capturing smooth extrema without clipping. Kim and Kim [29] proposed a MLP (Multi-dimensional Limiting Process) method which demonstrates a fine feature of controlling

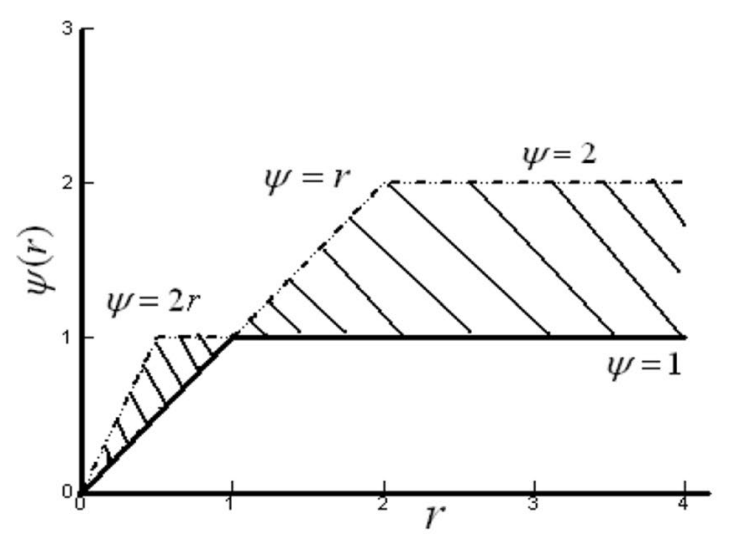

(a) Sweby's TVD limiter region numerical oscillations in multi-space dimensions with very desirable properties in terms of accuracy, efficiency and robustness. Yoon and Kim [30] modified the aforementioned MLP and refined it for three-dimensional applications without assuming local gradients, which made an excellent improvement on the solution accuracy, convergence, as well as the robustness for the steady/unsteady flows.

Although much effort has been invested in developing and testing different kinds of limiters, there is yet no widely accepted unambiguous conclusion on their attributes. Scott [31] first performed a systematical investigation on the limiter functions in MUSCL-based upwind schemes. Among the investigated limiters, van Albada's limiter was considered as the most attractive one because of its less compressive properties and less limitation on time marching step, whereas Min-mod limiter was considered as the most dissipative one with the least accurate prediction of discontinuities. Scott's conclusion on the investigated limiters has been widely accepted in the current practice of CFD. However, the conclusion on Min-mod limiter is still incomplete. The authors, in this paper, argue that Min-mod type limiter has various forms and its properties are actually determined by a special compression parameter (as described later). In Ref. [31], Scott's discussions only referred to one situation of Min-mod-type limiter's properties, and the others with different compression parameters were not discussed at all. After that, there has been no relevant and compelling literature to study this issue in depth and in detail. Although Min-mod limiter is considered to be the most dissipative limiter in general, it is widely accepted in CFD practice and embedded in various in-house CFD codes and commercial CFD software packages, and surprisingly demonstrating its capability of achieving acceptable numerical results with robustness. Very few researchers [32,33] are aware of this inconsistent behaviour of Min-mod-type limiter of being a dissipative limiter with more accurate predictions of flow

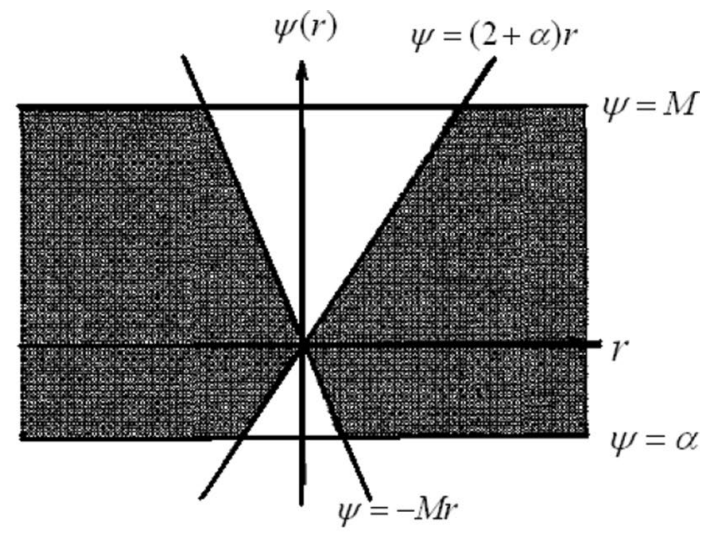

(b) Spekreijse's monotonic limiter region

Fig. 1 Second-order limiter region. a Sweby's TVD limiter region, b Spekreijse's monotonic limiter region 
discontinuities. A thorough understanding of its underlying reasons has never been explored.

As noted by Scott [31], the limiter function in MUSCLbased upwind schemes plays important roles on the numerical solutions of the shockwave-containing flows. Section 2 presents the in-house-CFD code developed by the authors, modifications made by the authors to the MUSCL interpolation through the incorporation of Spekrejise's primary modifications to the limiter functions are presented in detail in Sect. 3. The properties of several frequently utilized limiters [34] as listed in Sect. 3 are discussed systematically based on Sweby's TVD limiter region and Spekreijse's monotonic limiter region. In Sect. 4 a, hybrid-order estimator based on grid-independent theory is introduced, and it is used to perform the current investigation of limiter functions' properties. Following in Sect. 5, the numerical solutions from various limiters are discussed in detail. Finally, a general conclusion is drawn on the properties of commonly used limiter functions in the numerical simulation of the flows containing shockwaves, in particular on Min-mod-type limiter's compressive properties which have never been referred to or adequately investigated in previous literature because of the historic overemphasis of it being the most dissipative limiter. All the numerical test cases in this work are performed based on the calorically perfect gas model with a constant specific heat of 1.4.

\section{Numerical method}

The two-dimensional governing equations of Navier-Stokes flows are presented in the numerical computation domain $(\xi, \eta)$ as following:

$\frac{\partial \tilde{Q}}{\partial t}+\frac{\partial\left(\tilde{F}-\tilde{F}_{v}\right)}{\partial \xi}+\frac{\partial\left(\tilde{G}-\tilde{G}_{v}\right)}{\partial \eta}=0$

where $\tilde{Q}$ are conservative flow variables. $\tilde{F}, \tilde{G}, \tilde{F}_{v}$ and $\tilde{G}_{v}$ are convective fluxes and viscous fluxes, respectively.

The CFD code ATTF (Analysis Toolkits for Transonic Flows) [35] developed by $\mathrm{Li}$ is implemented to perform the current limiter function investigation. In ATTF, the viscous fluxes are calculated with the second-order central difference due to its elliptic nature. Most of the widely accepted spatial discretization schemes are enclosed in ATTF, such as Jameson's central-difference scheme, Van Leer's flux vector splitting scheme (FVS), Roe's flux difference splitting schemes (FDS), as well as AUSM (Advection Upstream Splitting Method) series of schemes. Previous literature showed that the computational results would be significantly influenced by the different intrinsic dissipation and dispersion properties of the spatial schemes, even with the same limiter function used [31]. A common belief is that the
FVS-type scheme is robust in application, but with the loss of the accuracy in solutions due to the hefty numerical dissipation. To achieve the sufficient accuracy in solutions with FVS scheme, one has to use a more refined computational grid to decrease the computational dissipation caused by the insufficient accuracy of spatial discretization. Roe's FDS scheme is another popular choice for solving flow governing equations with high resolution and high fidelity, but there is a possibility of violating the entropy condition, which may lead to significant difficulties in simulating high-speed complex flows. In Ref. [36], AUSMpw+ was proposed as an improved AUSM type of scheme [37], which has the advantages of having not only the robustness of FVS schemes but also the high resolution and high accuracy of FDS schemes for CFD practical problems. Therefore, AUSMpw+ scheme is chosen to perform the aimed limiter function investigation in the current research.

\section{Review of limiter functions}

The original van Leer's MUSCL interpolation [21] is written as following:

$\left(q_{\mathrm{L}}\right)_{i+\frac{1}{2}}=q_{i}+\frac{1}{4}\left[(1-\kappa) \Delta_{-}+(1+\kappa) \Delta_{+}\right]_{i}$
$\left(q_{\mathrm{R}}\right)_{i+\frac{1}{2}}=q_{i+1}-\frac{1}{4}\left[(1-\kappa) \Delta_{+}+(1+\kappa) \Delta_{-}\right]_{i+1}$

where $q_{\mathrm{L}}$ and $q_{\mathrm{R}}$ are primitive flow variables at left and right sides of the grid cell faces, and

$\Delta_{+}=q_{i+1}-q_{i}, \Delta_{-}=q_{i}-q_{i-1}$

$\kappa$ is a parameter that determines the spatial accuracy. As $\kappa$ increases from -1 to $1 / 3$, the solution accuracy would increase and $\kappa=0$ and $\kappa=1 / 3$ are the commonly adopted options in practice.

It has been proven that the linear interpolation formulas used to obtain second-order accurate algorithm, like Eq. (2), are not adequate since they can cause unphysical oscillations in solutions where discontinuities exist. According to Spekreijse [25], Eq. (2) can be reformulated as:

$$
\begin{aligned}
& \left(q_{\mathrm{L}}\right)_{i+\frac{1}{2}}=q_{i}+\frac{1}{2} \psi\left(r_{i}\right)\left(\Delta_{-}\right)_{i} \\
& \left(q_{\mathrm{R}}\right)_{i+\frac{1}{2}}=q_{i+1}-\frac{1}{2} \psi\left(1 / r_{i+1}\right)\left(\Delta_{+}\right)_{i+1}
\end{aligned}
$$

where $\psi(r)$ is expressed as following:

$\psi(r)=\frac{1}{2}[(1-\kappa)+(1+\kappa) r] \phi(r)$

and 
$r=\Delta_{+} / \Delta_{-}$

In computing numerical fluxes, limiter function will result in a reduction in accuracy in discontinuous regions. Therefore, the original formulation of Eq. (2) should be retained in smooth regions. It can be done by setting $\phi(r)=1$ in Eq. (4), then Eqs. (2) and (3) are equivalents, which implies that, in smooth regions, limiter function can be switched off with $\phi(r)=1$.

Four commonly implemented limiter functions, as investigated by Sweby and Spekreijse, respectively [34], are summarized as following:

Superbee limiter: $\quad \psi(r)=\max [\min (2 r, 1), \min (r, 2), 0]$

van Leer limiter : $\quad \psi(r)=\frac{|r|+r}{1+r}$

van Albada limiter : $\quad \psi(r)=\frac{r^{2}+r}{1+r^{2}}$

Hemker-Koren limiter : $\quad \psi(r)=\frac{2 r^{2}+r}{2 r^{2}-r+2}$

The current research focuses on further characterizing Minmod-type limiter. However, there exist two different forms of Min-mod-type limiter in the literature, which are both different in mathematical expressions and intrinsic attributes.

The first form of Min-mod limiter discussed by Sweby [19] is expressed as:

Min - mod limiter : $\psi(r)=\max [\min (r, 1), 0]$

Scott [31] and Anderson [38] employed different forms of Min-mod limiter in their work, respectively, of which the characteristics are determined by a compression parameter. To make a completely transparent comparison between selected limiter functions, Min-mod limiter employed by Scott [31] and Anderson [38] is reformulated to have a similar form as that of Eq. (6).

The MUSCL interpolation formula used in $[31,37]$ is presented below:

$\left(q_{\mathrm{L}}\right)_{i+\frac{1}{2}}=q_{i}+\frac{1}{4}\left[(1-\kappa) \bar{\Delta}_{-}+(1+\kappa) \bar{\Delta}_{+}\right]_{i}$

$\left(q_{\mathrm{R}}\right)_{i+\frac{1}{2}}=q_{i+1}-\frac{1}{4}\left[(1-\kappa) \bar{\Delta}_{+}+(1+\kappa) \bar{\Delta}_{-}\right]_{i+1}$

where

$\bar{\Delta}_{-}=\min \bmod \left(\Delta_{-}, \beta \Delta_{+}\right)=\Delta_{-} \max [\min (1, \beta r), 0]$

$\bar{\Delta}_{+}=\min \bmod \left(\Delta_{+}, \beta \Delta_{-}\right)=\Delta_{-} \max [\min (r, \beta), 0]$

and $\beta$ are the compression parameters given by
$1 \leq \beta \leq \frac{3-\kappa}{1-\kappa}, \quad(\kappa \neq 1)$

In general, the maximum allowable value of $\beta$ is adopted.

By setting $r=\Delta_{+} / \Delta_{-}$, Eq. (7) can be reformulated in the form of Eq. (3), and then the corresponding Min-mod limiter function is written as:

$\psi(r)=\frac{1}{2}\left[(1-\kappa) \phi_{1}(r)+(1+\kappa) \phi_{2}(r)\right]$

where

$\phi_{1}(r)=\max [\min (1, \beta r), 0]$

$\phi_{2}(r)=\max [\min (r, \beta), 0]$

Equation (8) will produce several forms of Min-modtype limiter with different $\kappa$ and $\beta$ :

- if $\beta=1$, it is the same with Eq. (6)

$$
\psi(r)=\max [\min (r, 1), 0]= \begin{cases}1 & r \geq 1 \\ r & 0 \leq r \leq 1 \\ 0 & \text { else }\end{cases}
$$

- if $\kappa=-1$ and $\beta=(3-\kappa) /(1-\kappa)=2$

$\psi(r)= \begin{cases}2 r & 0 \leq r \leq 1 / 2 \\ 1 & r \geq 1 / 2 \\ 0 & \text { else }\end{cases}$

- if $\kappa=0$ and $\beta=(3-\kappa) /(1-\kappa)=3$

$\psi(r)= \begin{cases}2 r & 0 \leq r \leq 1 / 3 \\ (1+r) / 2 & 1 / 3 \leq r \leq 3 \\ 2 & r \geq 3 \\ 0 & \text { else }\end{cases}$

- if $\kappa=1 / 3$ and $\beta=(3-\kappa) /(1-\kappa)=4$

$$
\psi(r)= \begin{cases}2 r & 0 \leq r \leq 1 / 4 \\ (1+2 r) / 3 & 1 / 4 \leq r \leq 4 \\ 3 & r \geq 4 \\ 0 & \text { else }\end{cases}
$$

With the above modifications to Min-mod-type limiter, Eq. (7) can be expressed as a function of $\psi(r)$ as that of Eq. (3), which makes the comparisons among the different limiters listed in Eqs. (5) and (10) easier. All limiters given in Eqs. (5) (6) and (10) are plotted together in Fig. 2. Figure 2 highlights the fact that, except for Min-mod limiter of $\beta=4$, all others lie in Sweby's TVD limiter region (illustrated in Fig. 1a). Part of $\beta=4$ Min-mod limiter lies 
Fig. 2 Curves of limiter functions. a Limiters in Eqs. (5a)(5c) and (6), b Min-mod-type limiter and Hemker-Koren limiter

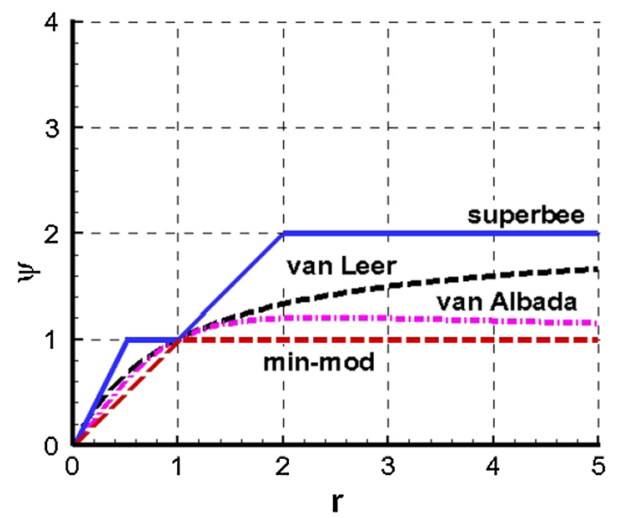

(a) Limiters in Eq.(5a)-(5c) and Eq.(6)

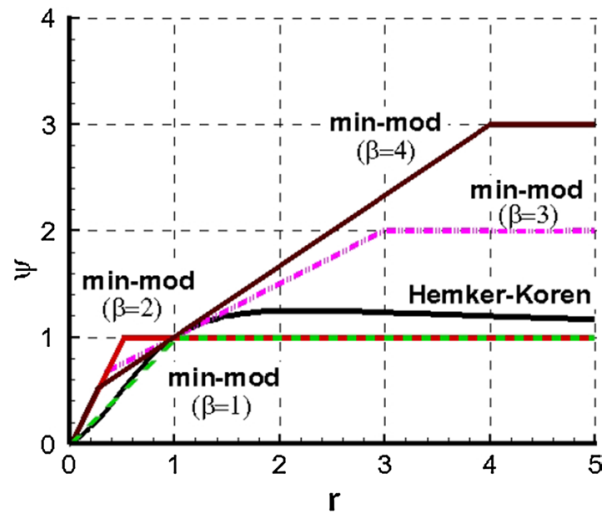

(b) Min-mod type limiter and Hemker-Koren limiter outside of Sweby's TVD region. According to Spekreijse's monotonic second-order region shown in Fig. 1b, i.e.

$\alpha \leq \psi(r) \leq M$

$-M \leq \frac{\psi(r)}{r} \leq 2+\alpha, \quad M \in(0, \infty)$ and $\alpha \in[-2,0]$

It is obvious that Min-mod-type limiter shown in Eq. (10d) really satisfies monotonicity-preserving condition by setting $M=3$ and $\alpha=0$, where $M$ and $\alpha$ are two parameters used to define Spekreijse's monotonic secondorder region.

In general, all limiters shown in Fig. 2a can roughly be classified into two categories: the ones close to the left and upper bound (Superbee limiter) of the TVD region are compressive limiters, which can achieve high-accuracy results, but possess unsound convergence behaviour and severe restrictions on time step size. Sweby [23] performed the detailed investigation on Superbee's properties and showed that Superbee limiter is an extremely compressive limiter which could give remarkably sharp profiles for the linear and nonlinear advection problem. However, the ones close to the right and lower bound (Mid-mod limiter of $\beta=1$ ) are dissipative limiters, which show robust and well-converged behaviour, but produce low-accuracy results in general. In Fig. 2a, all limiter functions shown in Eqs. (5a)-(5d) and (6) are in the sequence of increasing dissipation and decreasing compression, which is similar to the trend observed in CFD practice. However, this does not always hold true for Minmod-type limiters shown in Fig. 2b, which clearly indicates that the properties of Min-mod-type limiter are determined by the compression parameter $\beta$ defined in Eq. (10). Scott [31] only discussed one case of Min-mod limiter shown in Eq. (6) and/or Eq. (10a) with $\beta=1$. However, with $\beta=3$ or $\beta=4$, Fig. $2 \mathrm{~b}$ demonstrates that Min-mod limiter is more compressive than van Leer limiter. If $\beta=2$, Min-mod limiter is compressive in the region of $r<1$, equivalent to Superbee limiter, but dissipative in the region of $r>1$, equivalent to the case of $\beta=1$ Min-mod limiter. Therefore, it would be an incomplete conclusion to take Min-mod limiter as the most dissipative limiter without investigating its compressive features. The compressive behaviours of Min-mod-type limiter in practical applications will be further explored through three shockwave-containing flows in the following sections. One crucial aspect to be noted for Superbee limiter is that, it tends to turn smooth waves into square waves and makes the gradient sharper in solving practical flow. The overly compressive nature of Superbee limiter in multiple dimensions may lead to stair-casing effects at flow discontinuities. Due to its impracticality in engineering, Superbee limiter will not be discussed in the following sections. Min-mod limiter of $\beta=2$ will also not be considered because of its Superbeelike features in the region of $r<1$ as shown in Fig. $2 b$.

\section{Grid-convergence error analysis method}

There has been consensus on the effect of limiters on the convergence properties and accuracy of numerical solutions. However, most of the existing literature is based on the conclusion of qualitative analysis, and it is difficult to provide quantitative analysis results for the characteristics of different limiters and their comparison due to the lack of effective error analysis tools. For SWBLI problem, the general numerical solutions based on upwind schemes can only achieve up to third-order accuracy due to the existence of shockwave discontinuity in the flow field, and even only the first order can be reached at the discontinuity. Roach $[39,40]$ proposed a global numerical error analysis method based on Richardson-extrapolation theory, but the effectivity on the discontinuity-containing flow field error analysis was less than expected. Roy et al. [41-43] demonstrated that the first-order and second-order errors coexist in the numerical solutions of discontinuity-containing flows, and some 
flow quantities are non-monotonically converged with the grid refinement. The occurrence of non-monotonic gridconvergence solution is due to the opposite sign of the firstand second-order errors. Therefore, Roy [41] proposed a hybrid-order error analysis method for this non-monotonic grid-convergence phenomenon in discontinuity-containing flows. In this research, the same hybrid-order error analysis method by Roy is adopted to evaluate the performance of selected limiter functions.

The hybrid-order analysis method by Roy [41] is expressed as the following Eq. (12).

$f_{1}=f_{\text {exact }}+g_{1} h_{1}+g_{2} h_{1}^{2}+O\left(h_{1}^{3}\right)$
$f_{2}=f_{\text {exact }}+g_{1} h_{2}+g_{2} h_{2}^{2}+O\left(h_{2}^{3}\right)$
$f_{3}=f_{\text {exact }}+g_{1} h_{3}+g_{2} h_{3}^{2}+O\left(h_{3}^{3}\right)$

where $f_{k}$ is the flow quantity on the $k$ th level grid, and the densest grid is indicated as $k+1$. $f_{\text {exact }}$ is defined as the exact solution of flow quantities. The grid size scaling factor of $k+1$ th grid to $k$ th grid is defined as

$r_{k, k+1}=h_{k+1} / h_{k}$

The detailed information of other symbols, such as $g_{i}$ and $h_{k}$, can be found in [32].

By setting the grid scaling factor shown in Eq. (13) as a constant, the approximate solution of $g_{1}, g_{2}$ and $f_{\text {exact }}$ can be obtained from Eq. (12), which is expressed as following:

$\tilde{g}_{1}=\frac{r^{2} \varepsilon_{21}-\varepsilon_{32}}{r(r-1)^{2}}$

$\tilde{g}_{2}=\frac{\varepsilon_{32}-r \varepsilon_{21}}{r(r+1)(r-1)^{2}}$

$\tilde{f}_{\text {exact }}=f_{1}+\frac{\varepsilon_{32}-\left(r^{2}+r-1\right) \varepsilon_{21}}{(r+1)(r-1)^{2}}$

where

$\varepsilon_{32}=f_{3}-f_{2}, \quad \varepsilon_{21}=f_{2}-f_{1}$

The approximate solution of $\tilde{f}_{\text {exact }}$ shown in Eq. (16) is generally third-order accurate, and the spatial discretization error on the $k$ th grid with respect to $\tilde{f}_{\text {exact }}$ can be expressed as following:

$\mid$ spatial error $(\%)|=| \frac{f_{k}-\tilde{f}_{\text {exact }}}{\tilde{f}_{\text {exact }}} \mid \times 100$

Based on Roy's hybrid-order error analysis method, the first- and second-order errors can be given as follows, and the sum of them is presented at the same time in the following formulation: $\left|\frac{\tilde{g}_{1} h}{\tilde{f}_{\text {exact }}}\right| \times 100$

$\left|\frac{\tilde{g}_{2} h^{2}}{\tilde{f}_{\text {exact }}}\right| \times 100$

$\left|\frac{\tilde{g}_{1} h-\tilde{g}_{2} h^{2}}{\tilde{f}_{\text {exact }}}\right| \times 100$

\section{Results and discussion}

\subsection{NACA 0012 airfoil}

Anderson [38] studied the properties of Min-mod limiter of $\beta=4$ and the differentiable Albada-like limiter in twodimensional transonic Euler flow around NACA 0012 airfoil. With the same 2D case in the present work, a series of computational grids are generated to perform the gridindependence investigation. The free stream flow parameters are given as $\mathrm{Ma}_{\infty}=0.8, \alpha=1.25^{\circ}$ Although there is special interest in SWBLI problems in the present work, it is a common way to verify the characteristics of a spatial scheme by performing Euler-based numerical simulations, and the same idea is adopted in verifying the limiter functions' properties in this test.

Table 1 provides the detailed grid descriptions of the grid dimensions and the grid spacing. Figure 3 shows the drag coefficients calculated by different limiters on all grids, and the Richardson-extrapolation estimated drag coefficients on $h=0$ grid. It can also be concluded that, with grid refinement, the computed drag coefficients could be grid-converged in the sense of Richardson-extrapolated estimate, and the estimated drag coefficients obtained by Eq. (16) for different limiter functions are slightly different. The varying of estimated flow quantities indicates that the numerical prediction accuracy is related to the intrinsic properties of the limiter function embedded in the numerical scheme.

Table 1 Computational grids information

\begin{tabular}{lcl}
\hline Grid number & Grid points & $\begin{array}{l}\text { Grid } \\
\text { spacing } \\
h^{\mathrm{a}}\end{array}$ \\
\hline 1 & $641 \times 257$ & 1 \\
2 & $321 \times 129$ & 2 \\
3 & $161 \times 65$ & 4 \\
4 & $81 \times 33$ & 8 \\
\hline
\end{tabular}

${ }^{\mathrm{a}}$ Grid spacing measure is normalized by the grid spacing on the finest grid (e.g. grid 1 has $h=1$ ) 


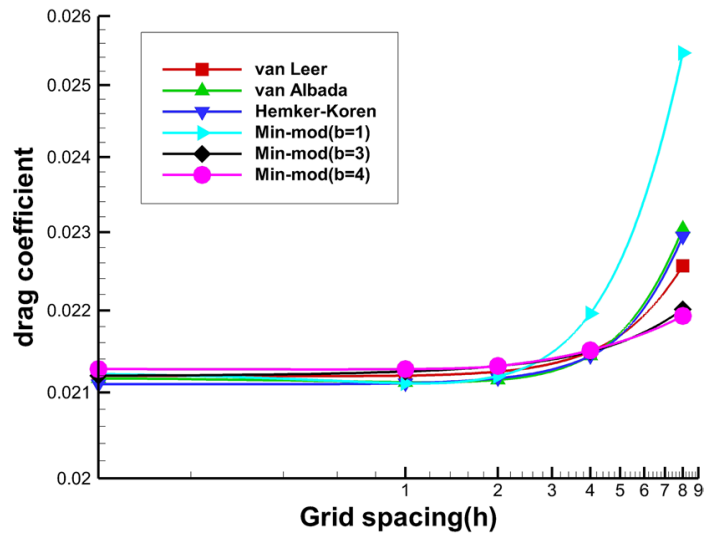

Fig. 3 Comparison of drag coefficients by different limiter functions
Table 2 presents the Richardson-extrapolation estimate of drag coefficients on $h=0$ grid, the numerically predicted drag coefficients on $h=1$ grid for different limiter functions. It can be obviously seen that the Richardson-extrapolation estimate of drag coefficient value for Min-mod limiter of $\beta=4$ is the highest, while for Hemker-Koren limiter is the lowest. The estimated hybrid-order spatial errors by Eq. (17) for different limiter functions on $h=1$ grid are also shown in Table 2, which indicates that the spatial discretization error from Min-mod limiter of $\beta=4$ is the least, while $\beta=1$ is the largest.

Figure 4 shows the curves of spatial errors for different limiter functions as the grid refinement. The first- and second-order errors, as well as their sum, are calculated individually by Eq. (18a-18c). The discrete solution errors
Table 2 Comparison of hybridorder spatial errors for different limiter functions

\begin{tabular}{llll}
\hline Limiter functions & $\begin{array}{l}\text { Estimated exact drag coef- } \\
\text { ficient }(h=0)\end{array}$ & $\begin{array}{l}\text { Calculated drag coefficient } \\
(h=1)\end{array}$ & Spatial error (\%) \\
\hline van Leer & 0.021199 & 0.021201 & 0.005346 \\
van Albada & 0.021168 & 0.021121 & 0.224705 \\
Hemker-Koren & 0.021098 & 0.021110 & 0.054191 \\
Min-mod $(\beta=1)$ & 0.021221 & 0.021110 & 0.525733 \\
Min-mod $(\beta=3)$ & 0.021200 & 0.021251 & 0.240091 \\
Min-mod $(\beta=4)$ & 0.021280 & 0.021279 & 0.002036 \\
\hline
\end{tabular}

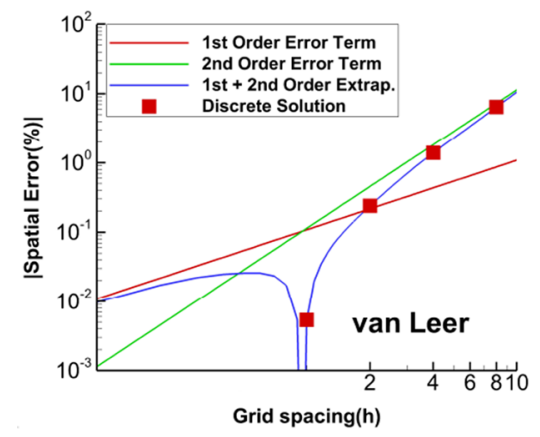

(a)

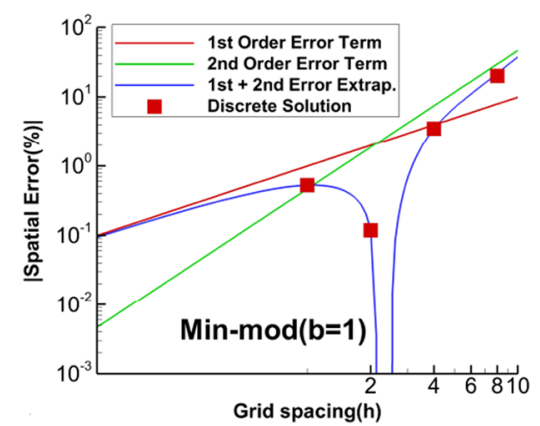

(d)

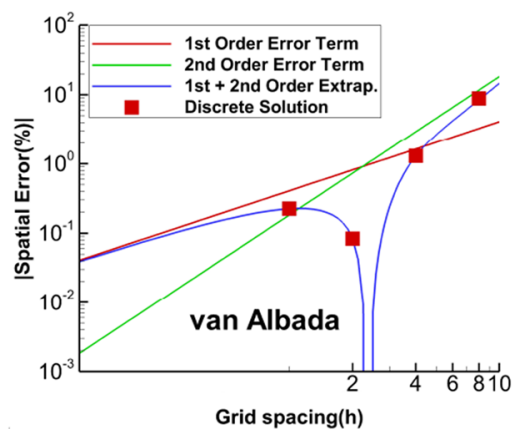

(b)

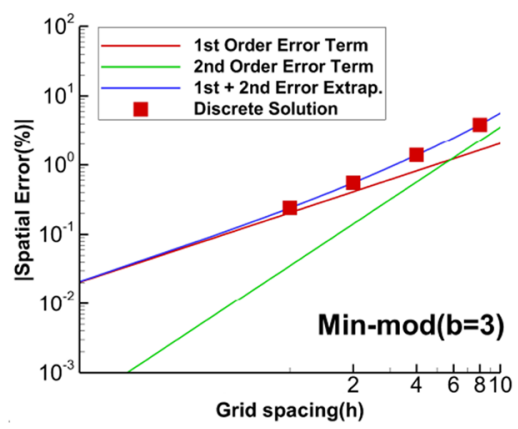

(e)

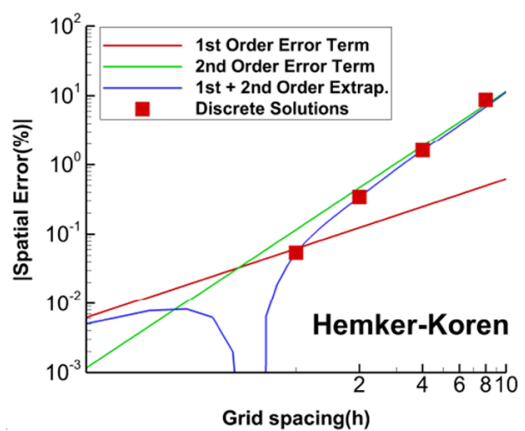

(c)

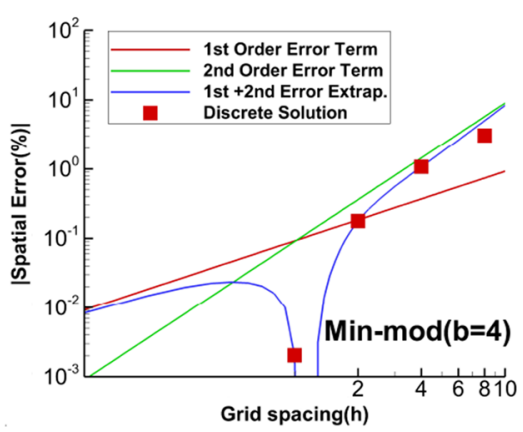

(f)

Fig. 4 Spatial errors for different limiter functions with grid refinement 
indicated by the square symbols in Fig. 4 are calculated from Eq. (17) where the approximate solution of $\tilde{f}_{\text {exact }}$ is from Eq. (16) using 1-3 grids. For all limiter functions, only the sums of the first- and second-order error terms on 1-3 grids coincide exactly with the discrete solution errors. The reason is that only $1-3$ grids are used to determine the coefficients in Eqs. (14) and (15). It can also be clearly seen from Fig. 4 that, except for Min-mod limiter of $\beta=3$, all other limiter functions capture the non-monotonic grid-convergence phenomenon that occurs with the grid refinement. The main reason for this phenomenon is that the first- and second-order errors with opposite signs would cancel each other out. Regardless of whether the grid convergence is monotonic or non-monotonic, the spatial error of different limiter functions on the coarse grid is gradually approaching the second-order accuracy, whereas on the fine grid it approaches the first-order range asymptotically, that means the solution accuracy of the focused shockwave-containing flows is between the first- and second-order accuracy.

Figure 5 plots the extrapolated sums of the first- and second-order errors of different limiters. It is very clear that, for the extrapolated spatial errors on the infinitely fine grid, Hemker-Koren limiter presents the lowest spatial discrete error, while Min-mod limiter of $\beta=1$ presents the highest spatial discrete error. Although with less dissipation level, Min-mod limiters of $\beta=3$ and $\beta=4$ do not show the less spatial discrete error than Hemker-Koren limiter. The main reason is that the contributions of the converged iterative error due to the differentiable limiter function, such as Hemker-Koren limiter, has to be taken into account.

Figure 6 presents the predicted pressure coefficients along the solid wall of the airfoil with the finest grid. All limiters yield similar pressure distributions over the almost whole part of the airfoil, and Min-mod limiter of $\beta=4$ captures the shockwave with the highest resolution on the upper

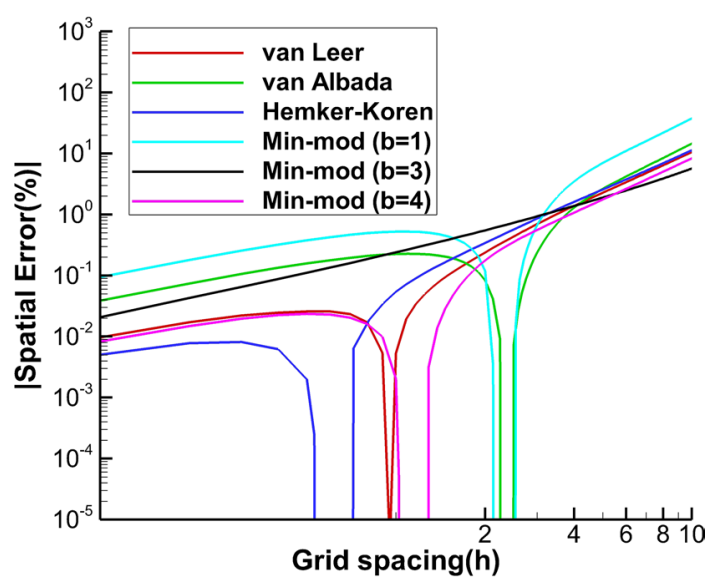

Fig. 5 Comparison of hybrid-order spatial errors for NACA 0012 airfoil

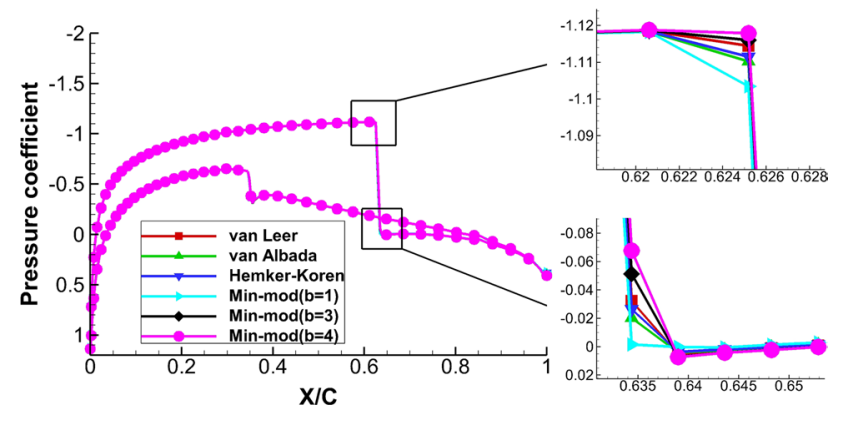

Fig. 6 Comparison of pressure coefficients for NACA 0012 airfoil

surface. However, the $\beta=1$ Min-mod limiter captures the shockwave with the lowest resolution. For the shockwave on the lower surface, there is a slight and sharp decrease on the pressure distributions after the shockwave (with a closer view not shown here). We believe that it is due to the slight increase in the velocity at the local position after the shockwave, and then the pressure begins to increase gradually along the lower surface. The results from the differentiable limiters are similar because of their similar dissipation levels. Although the pressure distributions along the airfoil surface for different limiters have minute differences when the finest computational grid is adopted, the influence of the inherent dissipative characteristics of the limiters can still be perceived in Fig. 6.

Another important aspect that may affect the computational solution accuracy is the iterative convergence error in numerical simulations. Venkatakrishnan [27] discussed the influences of limiters on converging to steady solutions of numerical simulation in great detail, pointed out that a nondifferentiable limiter might more severely hamper the convergence process of a numerical simulation and result in less accurate solutions than that of a differentiable limiter. Figure 7 shows the residual convergence process with respect to iteration steps for all abovementioned limiters on the densest grid and clearly demonstrates that the three differentiable limiters, i.e. van Leer limiter, Hemker-Koren limiter and van Albada limiter, exhibit excellent convergence performance by approaching to the machine zero level. However, as depicted in [38], the residuals of non-differentiable Minmod-type limiters fail to approach machine zero and exhibit numerical oscillations after a few orders of magnitude drops. Anderson et al. [38] also pointed out that the limit cycle oscillation in the iterative process for Min-mod limiter of $\beta=4$ was mainly due to its non-differentiable feature. Also, Ventakarishnan [27] concluded that the convergence behaviour is even worse in the case of non-differentiable limiter functions compared with that of smoothing and differentiable limiter functions.

The discussion mentioned above clearly demonstrates that conclusions from Fig. 7 are consistent with those in 


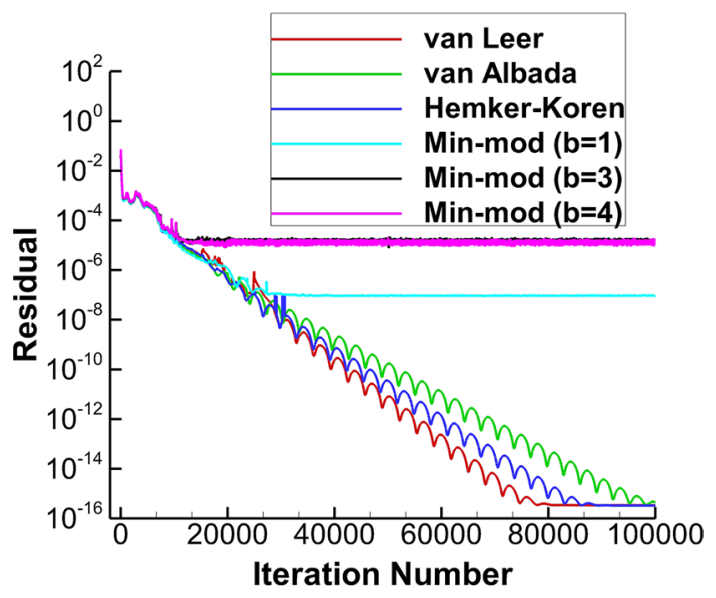

Fig. 7 Comparison of iterative residual histories for NACA 0012 airfoil

$[27,38]$, i.e. the non-differentiable limiter might severely deteriorate the convergence process of numerical simulations. The contrast that the compressive Min-mod limiters with $\beta=3$ and $\beta=4$ produce larger spatial errors than that of the less compressive Hemker-Koren limiter, as shown in Fig. 4, mainly comes from the poorly converged iterative residual due to the non-differentiable feature of Min-modtype limiter. The current study also demonstrates that, with a reasonable compression parameter $\beta$, Min-mod limiter could behave as a compressive limiter predicting the solution accurately with small spatial errors. Meanwhile, it might also become a dissipative limiter resulting in significant spatial errors, which correlate with the theoretical analysis shown in Fig. 2.

\subsection{Supersonic SWBLI on flat plate}

Hakkinen et al. [44] conducted an experiment on the laminar boundary layer flow interacting with an incident shock on a flat plate, as illustrated in Fig. 8. This experiment has been frequently used as a benchmark to verify various numerical algorithms. Here this same case is selected to perform the numerical evaluation of limiter functions.

The computational domain is defined as a 2-D rectangular with a length of $2 L$ and a width of $L$, and the reference length $L$ is defined as the distance from the leading edge of the plate to the impacting point of the incident shock on the plate. The free stream conditions are listed as following:

$$
\begin{aligned}
& \mathrm{Ma}_{\infty}=2.0, T_{\infty}=117 \mathrm{~K}, \mathrm{Re}_{\infty}=2.96 \times 10^{5}(\text { based on } L) \\
& \text { and } \beta=32.6^{\circ} \text { (incident shock angle). }
\end{aligned}
$$

For the numerical simulation, the left side of the rectangular domain is a supersonic inflow boundary with primitive variables being specified. The left-side inflow boundary is divided into two parts: the first part is below the incident point with the upstream condition of the incident shock being specified, and the second part is above the incident point with the downstream condition of the incident shock being specified. The downstream condition of the incident shock is calculated with Rankine-Hugoniot relationship, while the upstream condition of the incident shock is from the free stream condition, i.e. $\mathrm{Ma}_{\infty}=2.0$. The right side of the rectangular domain is an outflow boundary, which is far enough from the induced separating point so that a zeroorder extrapolation of the primitive variables in streamwise direction can be employed. The bottom of the rectangular domain is a no-slip solid wall on the surface of the plate with adiabatic temperature condition specified. The top side of the rectangular domain is defined as the downstream condition of the incident shock, the same as the upper part of the left-side inflow boundary. The same case has been studied in [32], and only the main results and conclusions are reviewed briefly here.

Table 3 describes the detailed information of the computational grids for the current study. Figure 9 presents the calculated drag coefficients by different limiter functions on all grids, and the Richardson-extrapolation estimate of drag values by Eq. (16) is also plotted. For different limiter
Fig. 8 Schematic of SWBLI on the flat plate

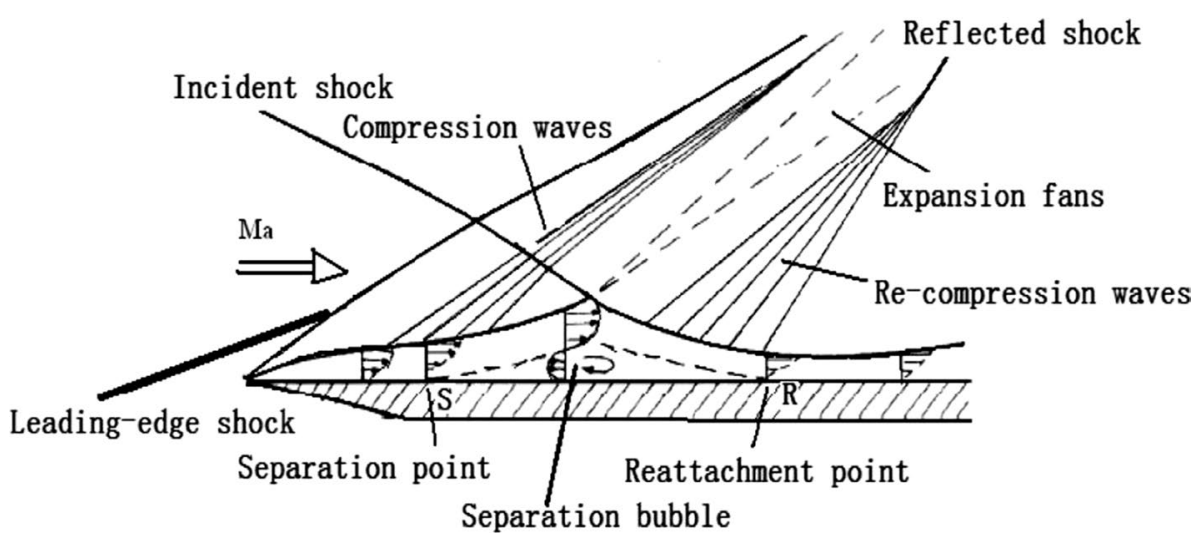


Table 3 Computational grids information

\begin{tabular}{lll}
\hline Grid number & Grid points & Grid spacing $h$ \\
\hline 1 & $401 \times 161$ & 1 \\
2 & $201 \times 81$ & 2 \\
3 & $101 \times 41$ & 4 \\
\hline
\end{tabular}

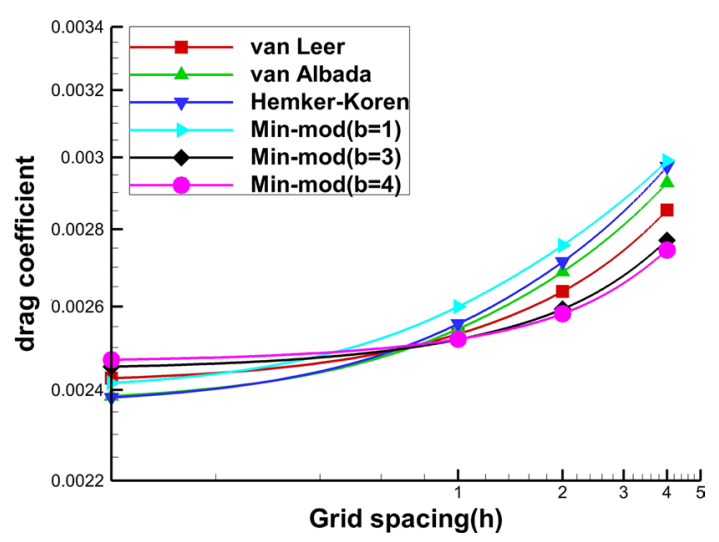

Fig. 9 Comparison of drag coefficients by different limiter functions on the flat plate

functions, the Richardson-extrapolation estimate of drag coefficients is slightly different, and the maximum difference between different limiters is about 1 count. The highest
Richardson-extrapolation estimate of drag is from Min-mod limiter of $\beta=4$, while the lowest is from Hemker-Koren limiter.

Figure 10 plots the curves of spatial errors for different limiters as the grid is refined. Although several limiter functions exhibit the non-monotonic grid-convergence features, the Richardson-extrapolation estimated solutions of different limiters approach to the second-order accuracy on the coarse grid, while to the first-order accuracy on the dense grid. Figure 11 presents the extrapolated hybrid-order spatial errors of different limiters. It can be clearly concluded that Min-mod limiter of $\beta=4$ is compressive, while $\beta=1$ is dissipative, because the solution accuracy from Min-mod limiter of $\beta=4$ is the highest, and is the lowest from $\beta=1$ Min-mod limiter.

A further investigation on the iterative convergence effects is performed on the currently densest grid, as shown in Fig. 12. Although Min-mod limiter of $\beta=1$ is non-differentiable, it still can converge to machine zero due to its inherent hefty dissipation, but with the lowest accuracy. For differentiable limiters, van Albada limiter and Hemker-Koren limiter can iteratively converge to machine zero, while van Leer limiter only drops a few orders in residuals. However, van Leer limiter still achieves the higher accuracy than van Albada and Hemker-Koren limiters do. Even with the unsound iterative convergence, Min-mod limiter of $\beta=4$ still achieves the lowest spatial errors. Although

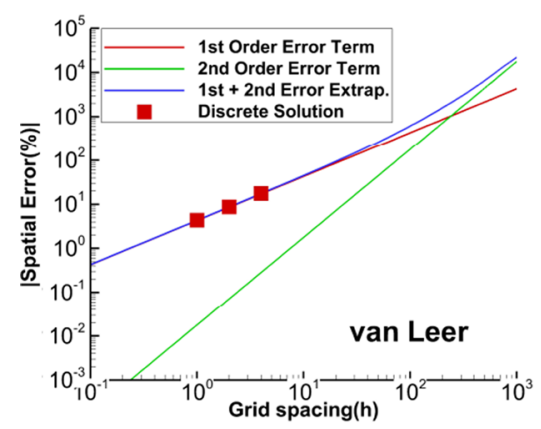

(a)

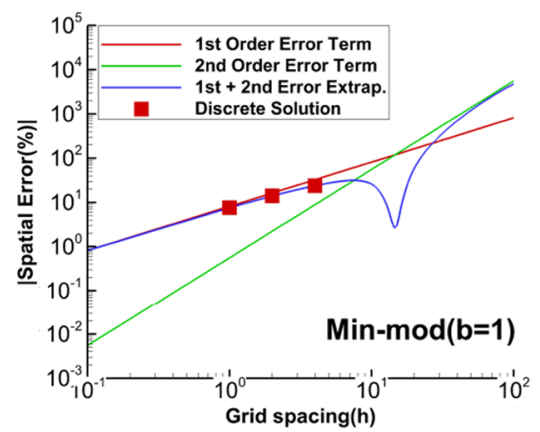

(d)

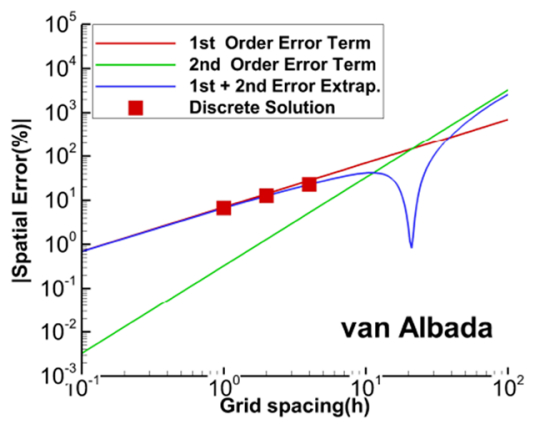

(b)

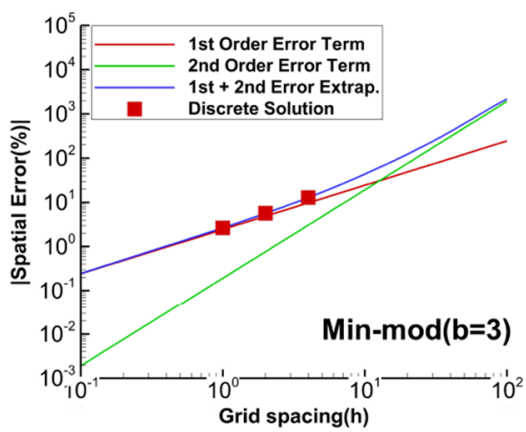

(e)

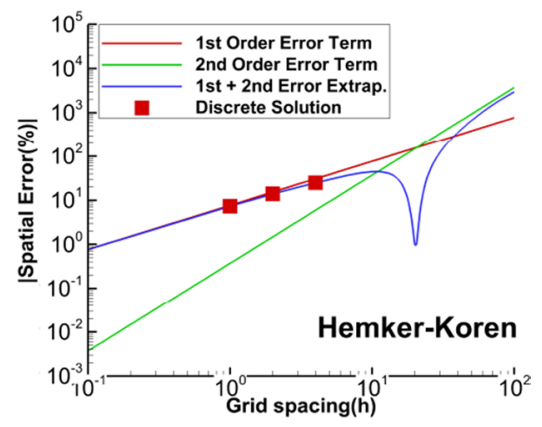

(c)

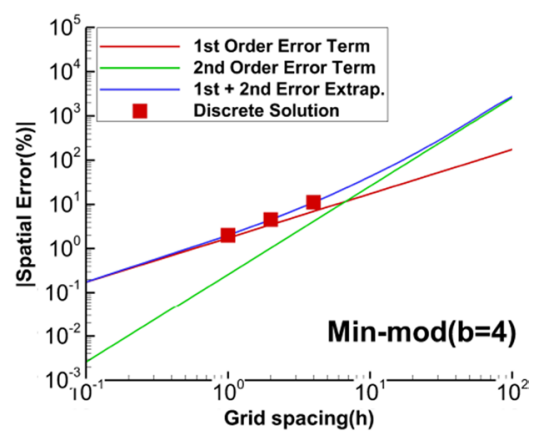

(f)

Fig. 10 Spatial errors for different limiter functions with grid refinement 


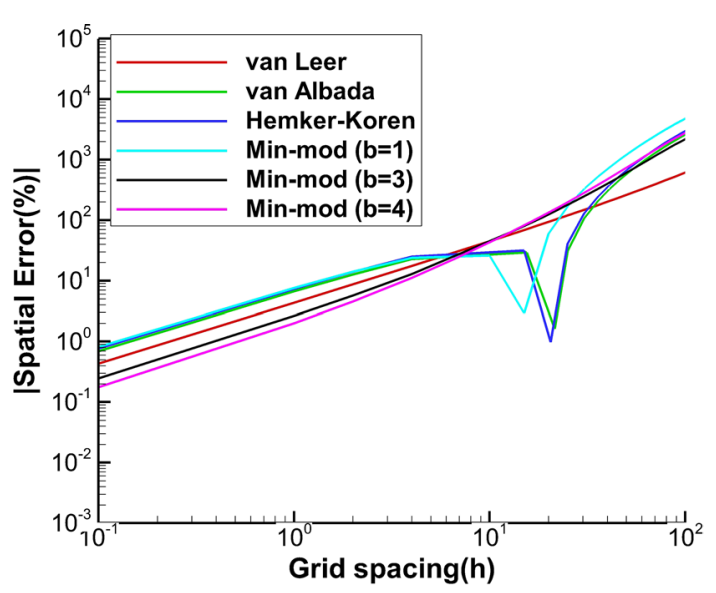

Fig. 11 Comparison of hybrid-order spatial errors for SWBLI on the flat plate

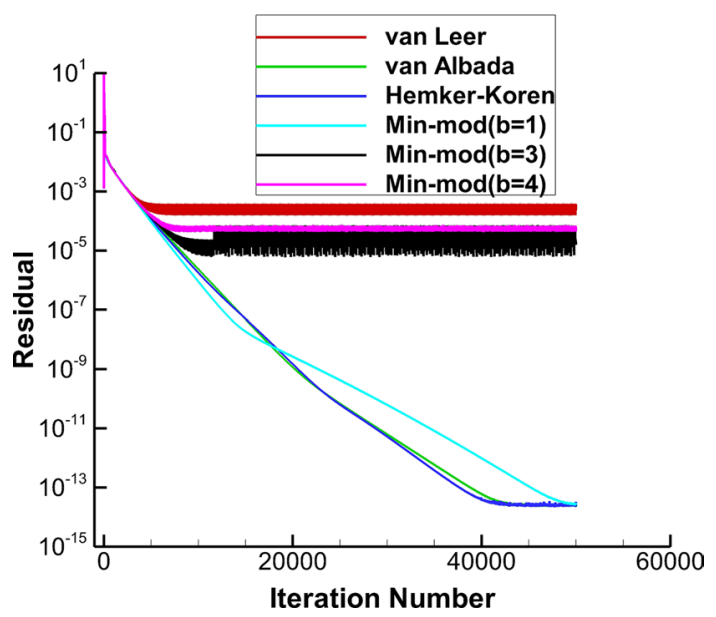

Fig. 12 Comparison of iterative residual histories for SWBLI on the flat plate

the differentiable or non-differentiable features of the limiter may influence the residual convergence, the dominating factor of the solution accuracy is the inherent dissipation of the employed limiter. Therefore, the difference in hybridorder spatial errors between different limiters mainly comes from the capabilities of capturing discontinuities, i.e. the dissipation level of limiters determines the accuracy of the numerical solution to a large extent.

A qualitative analysis of the concerned flow features is also performed. The contours of velocity divergence of different limiters on the densest grid are presented in Fig. 13. The whole field of flow structures, as shown in Fig. 8, are numerically captured clearly. Since the numerical simulation is obtained on the densest grid and is grid-converged in the sense of Richardson-extrapolated estimate, the discrepancy on flow structure resolutions between different limiters is rarely visible. According to the sequence of compressive limiters shown in Fig. 2 and corresponding conclusions of their properties, Min-mod limiter of $\beta=1$ with the most dissipation achieves the least accurate solutions, as illustrated in Fig. 13. It can be seen that, all of the waves, including the leading edge shockwave, the compression waves and the expansion waves, lose the high-resolution characteristics they deserve and are numerically smeared due to the hefty dissipation. In contrast, the results from Min-mod limiters of $\beta=3,4$ are of the higherorder shockwave resolutions than other limiter functions.

Figure 14 plots the computational results of different limiter functions on the densest grid compared with the experiment data on the flat plate. The differences between limiters are evident on the distribution of skin friction. Firstly, the positions of flow separation point, where the skin friction turns to the negative value, are of great discrepancy. Minmod limiter of $\beta=1$ with the most dissipation predicts the separation point at the most downstream position, while Minmod limiter of $\beta=3,4$ predicts the most upstream separation position. Secondly, the locations of flow reattachment point, where the skin friction turns back to the positive value, are predicted in a reversed order of the separation point. The reattachment point of $\beta=1 \mathrm{Min}-\bmod$ limiter is predicted at the most upstream, but $\beta=3,4$ Min-mod limiter is at the most downstream. Therefore, the extent of the separation region, which is induced by the impacting of incident shock with boundary layer, varies for different limiters. The compressive limiter function, like Min-mod limiter of $\beta=3,4$, predicts the largest separation bubble length, but the most dissipative limiter function, like Min-mod limiter of $\beta=1$, predicts the smallest separation bubble. The maximum difference of the predicted separation length is about $7.57 \%(0.03472 \mathrm{~L})$ according to the separation information shown in Table 4. Finally, the differences between the experimental results and the numerically predicted results with different limiters are of great discrepancy. As noted by Knight [4], the flow field of a SWBLI problem is dominated greatly by the unsteady shockwave system and flow separation, and the flowfield unsteadiness may explain the differences between RANS computations and experimental data when flow separation occurs.

Detailed information about the separation regions for different limiters is shown in Table 4 and illustrated in Fig. 14a. For the distribution of surface pressure shown in Fig. 14b, the primary discrepancy of different limiters is at the impacting point, where there is an obvious pressure plateau, which is determined by the size of the separation bubble. The larger the separate region is, the flatter the plateau of the pressure distribution is. Figure 15 shows the pictures of SWBLI separation bubble predicted by different limiters and the streamline spectrum in the vicinity. It can be seen that, the stronger the dissipation of the limiter, the smaller the predicted separation bubble size. The least dissipative Min-mod limiter of $\beta=3,4$ predicts the largest separation bubble size, which is consistent with 


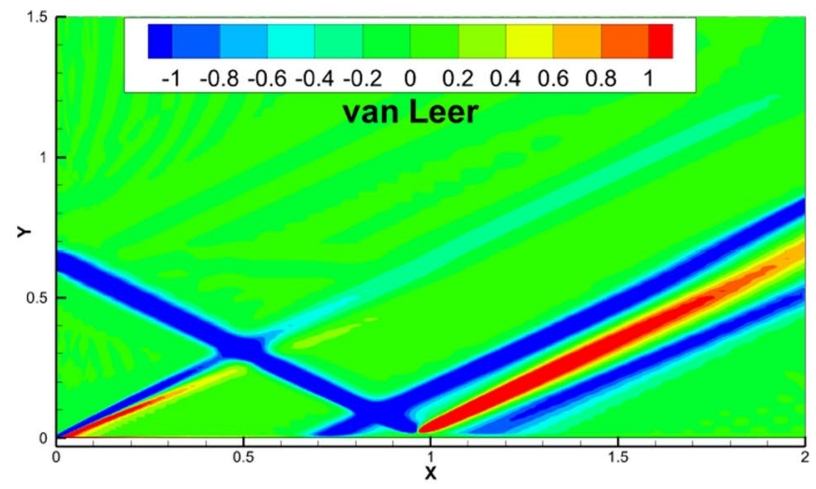

(a)

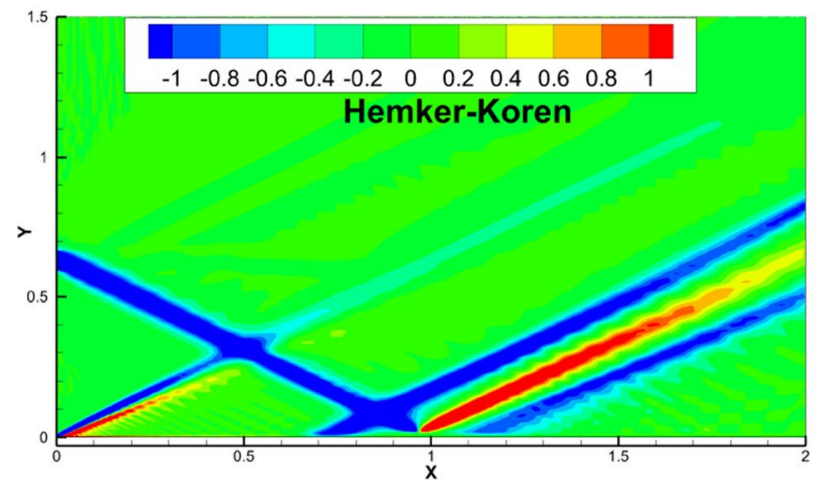

(c)

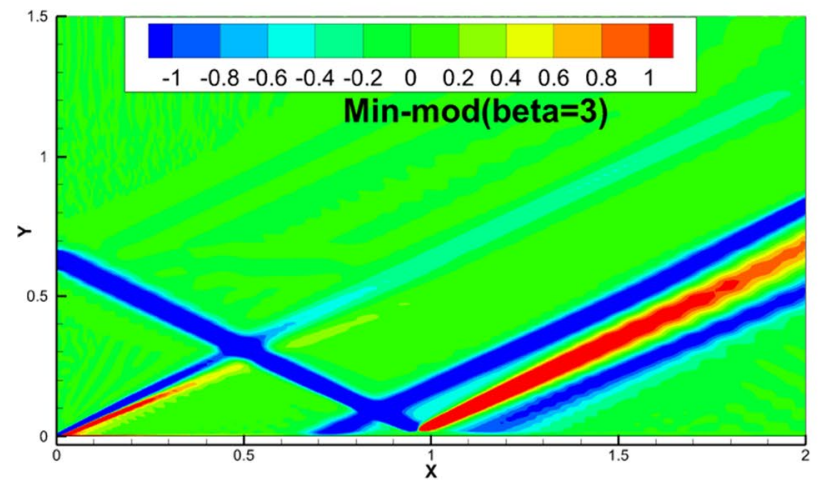

(e)

Fig. 13 Contours of velocity divergence for the flow over the flat plate

the separation bubble information given in Table 4. It is also stated that Min-mod limiter of $\beta=3,4$ is a compressive-type limiter with less dissipation.

\subsection{Hypersonic flow about a 24-deg compression ramp}

As presented schematically in Fig. 16, the current test is a complex hypersonic case with the strong interactions of shockwaves, expansion waves and boundary layer as well.

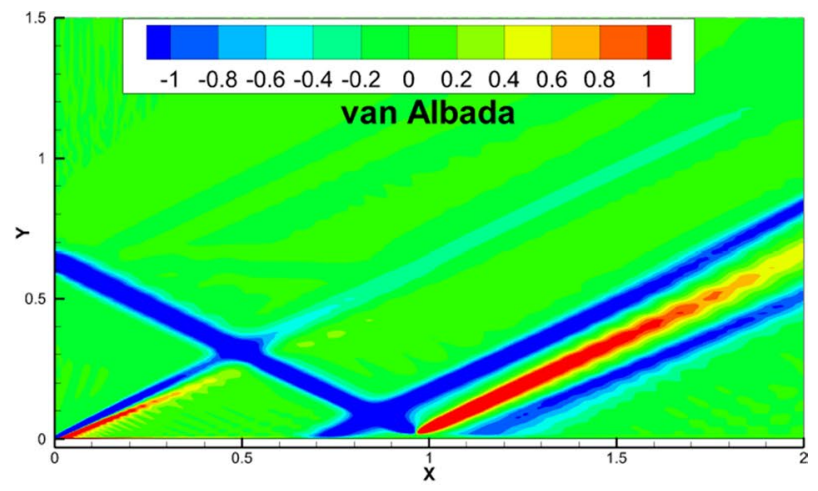

(b)

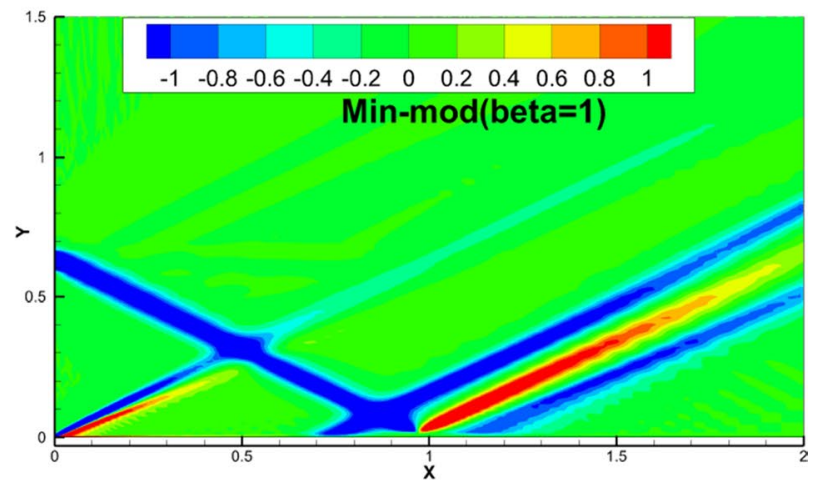

(d)

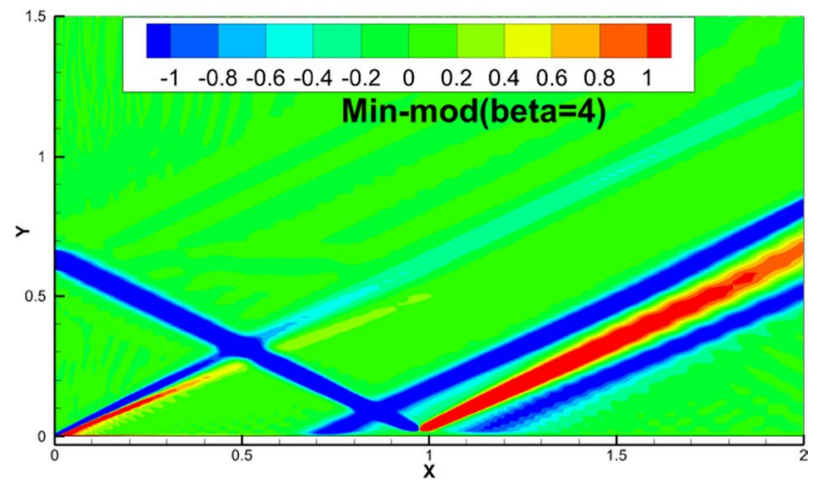

(f)

As shown in Fig. 16, a large recirculation region is formed in the $24^{\circ}$ corner of the compressive ramp. The free stream conditions are as follows, and the reference length $L$ is defined as the length of the horizontal plate:

$$
\begin{aligned}
& \mathrm{Ma}_{\infty}=14.1, T_{\infty}=72.7 \mathrm{~K}, \\
& T_{\text {wall }}=297 \mathrm{~K}, \mathrm{Re}_{\infty}=1.0369 \times 10^{5}
\end{aligned}
$$

(based on the reference length $L=1 \mathrm{~m}$ ) 
Fig. 14 Comparison of computed results. a Skin friction coefficient. b Normalized pressure
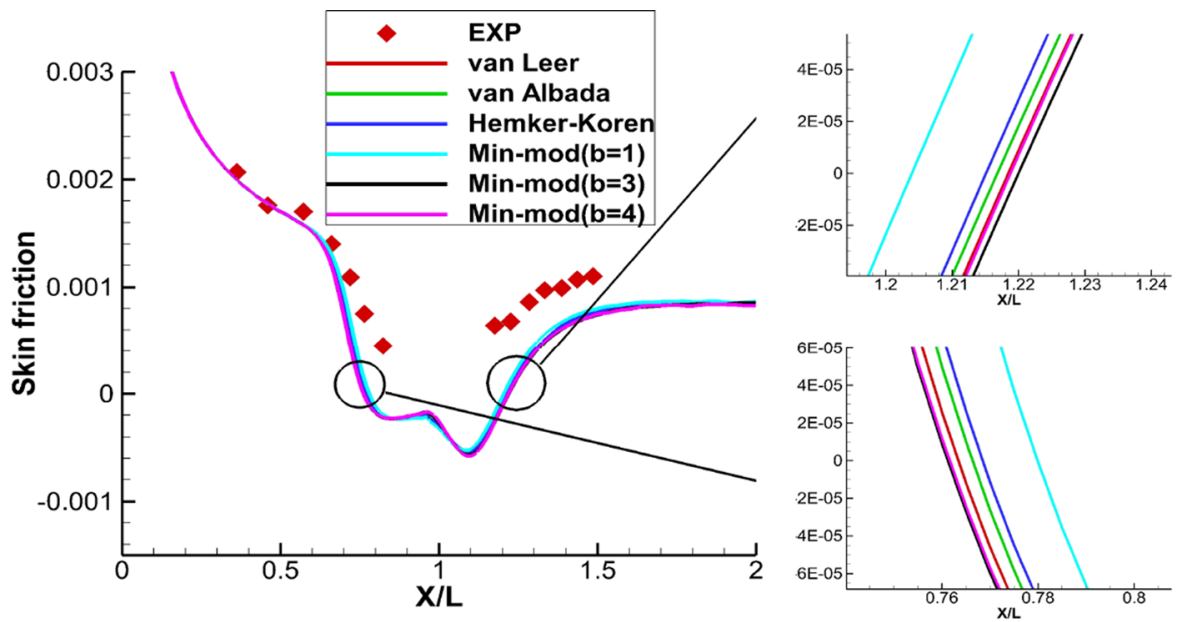

(a) Skin friction coefficient
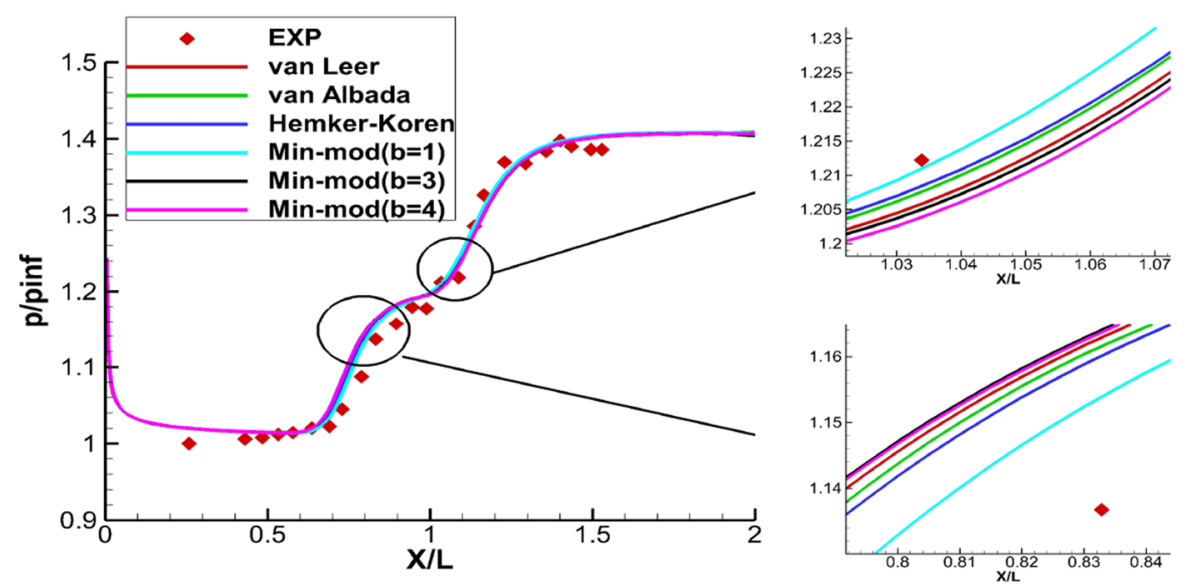

(b) Normalized pressure
Table 4 Detailed information of separation region for different limiters

\begin{tabular}{llll}
\hline Limiter function & $\begin{array}{l}\text { Separation } \\
\text { point }\left(X_{\mathrm{S}} / L\right)\end{array}$ & $\begin{array}{l}\text { Reattachment } \\
\text { point }\left(X_{\mathrm{R}} / L\right)\end{array}$ & Separation length \\
\hline van Leer & 0.76343 & 1.21851 & 0.45508 \\
van Albada & 0.76638 & 1.21685 & 0.45047 \\
Hemker-Koren & 0.76850 & 1.21516 & 0.44666 \\
Min-mod $\beta=1$ & 0.77988 & 1.20392 & 0.42404 \\
Min-mod $\beta=3$ & 0.76126 & 1.22002 & 0.45876 \\
Min-mod $\beta=4$ & 0.76164 & 1.21884 & 0.45720 \\
\hline
\end{tabular}

This case has viscous/inviscid interactions over a compression corner formed by the intersection of a flat plate and a wedge tested by Holden and Moselle [45]. The flow along the whole test section was completely laminar, thereby eliminating the issue of turbulence modelling in the current study due to the low Reynolds number. Even though the freestream Mach number was high, there were no significant real-gas effects because the freestream temperature was low. Rudy and Thomas [46] conducted a RANS (Reynolds Averaged Navier-Stokes equations) code validation study by performing a series of $2 \mathrm{D}$ high-speed laminar separated flow simulations for the SWBLI induced by the current compression ramp configure. The RANS method was adopted to explore the limiter functions' properties with this strong SWBLI of compression ramp flow in the current study. Although no RANS model is capable of accurate prediction of all aspects of the hypersonic compression ramp flow [1-5], the application of LES or DNS to compressible flows, SWBLI in particular, is very much in its infancy, and RANS method still plays important role in engineering, especially in configuration optimization problems with SWBLI phenomenon. Three computational grids were generated to perform the grid-independent study. For the densest grid, the grid point number on the horizontal plate is 161 , on the ramp plate is 241.161 grid points are distributed along the direction normal to the 


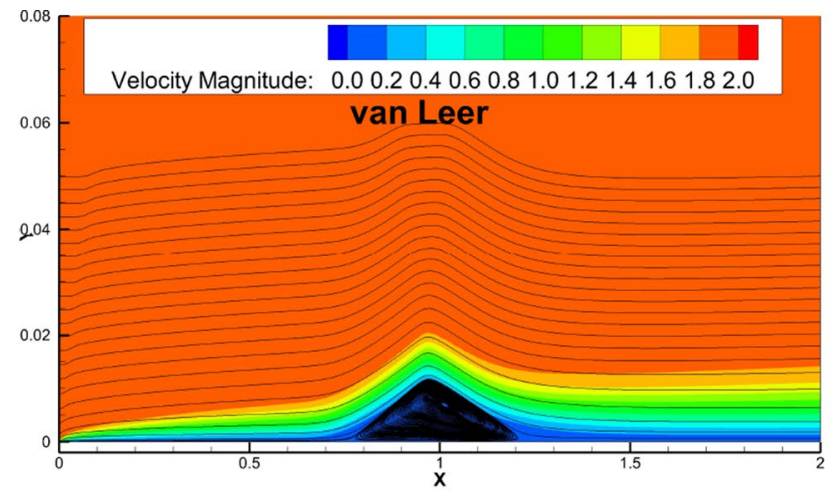

(a)

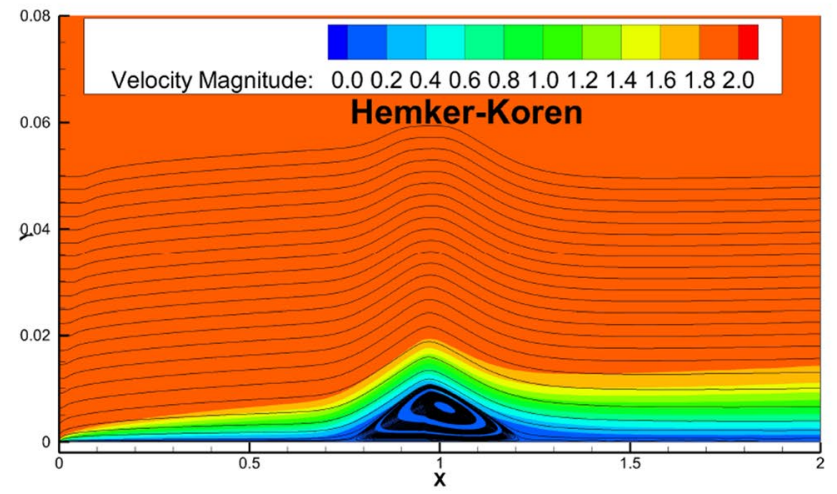

(c)

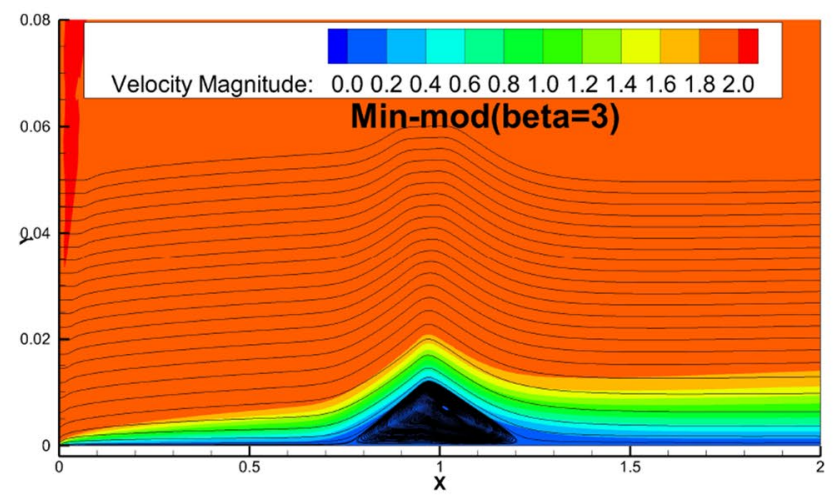

(e)

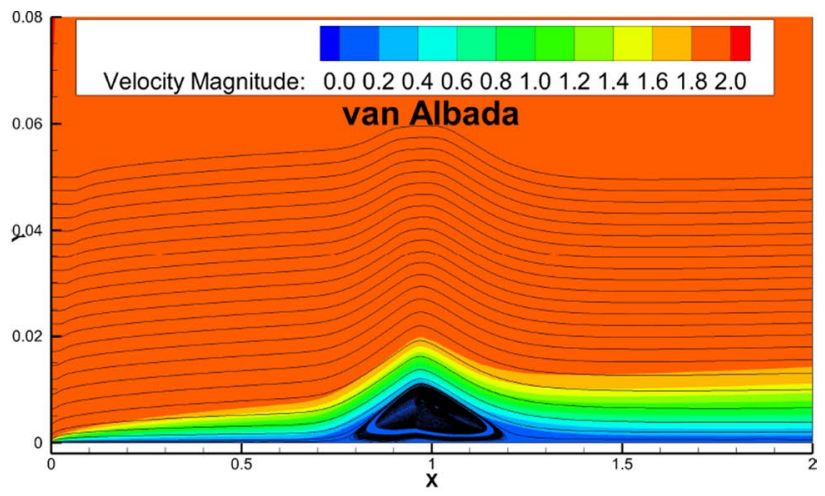

(b)

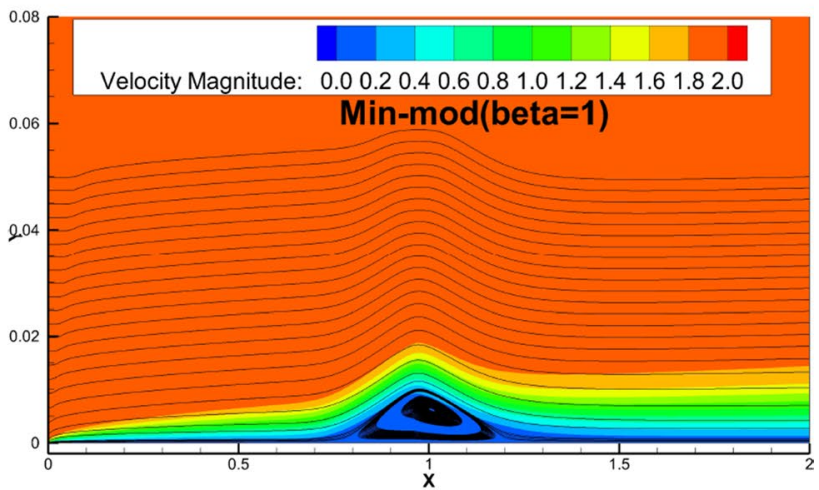

(d)

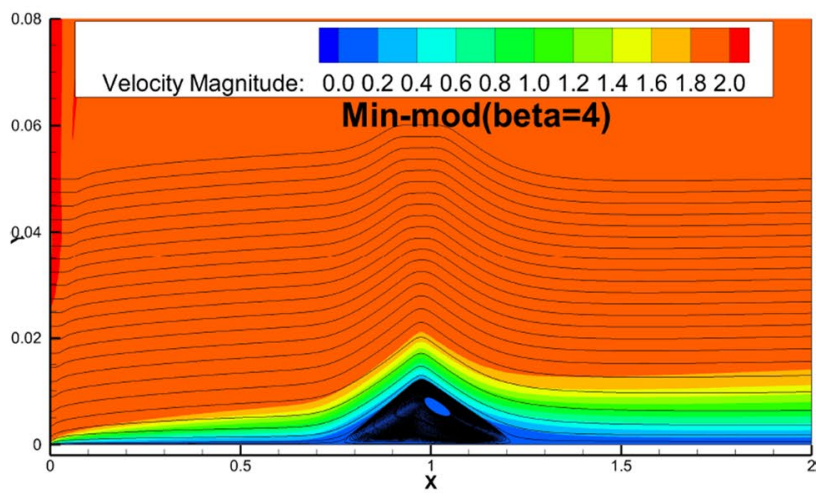

(f)

Fig. 15 Details of the streamline near separation region with different limiters

wall, with the first grid point distance is $2.0 \times 10^{-6} \mathrm{~L}$ off the solid wall.

Table 5 presents the detailed information of the computational grids. Figure 17 shows the comparison of the predicted drag coefficients on all grids with different limiters, which indicates the computations are grid-independent in the sense of Richardson-extrapolated estimate. The drag coefficients with Richardson-extrapolation method for all limiters are also plotted in Fig. 17. It can be concluded that, as the grid spacing approaches to zero, the predicted drag coefficients tend to converge to the Richardsonextrapolation estimated solutions for each limiter, respectively, but the estimated values are different for different limiters. The variation of the Richardson-extrapolation estimated solutions shown in the current test case, as well as in the aforementioned two test cases, indicates that the grid-converging process of different limiter functions is determined by their inherent dissipation properties. 


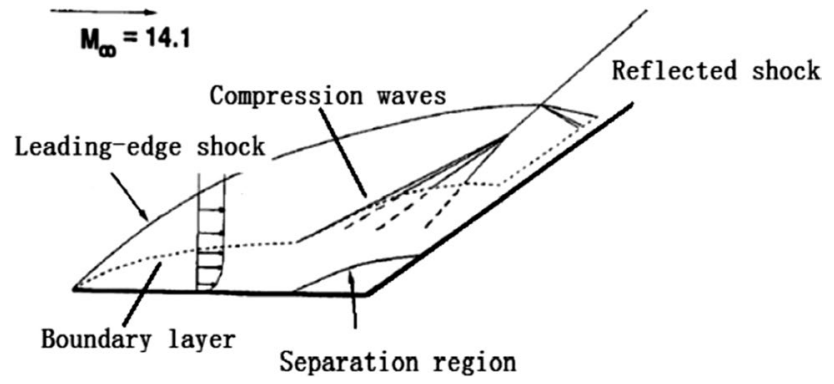

Fig. 16 Schematic of hypersonic flow past $24^{\circ}$ compression ramp

Table 5 Computational grid information (2D)

\begin{tabular}{lll}
\hline Grid number & Grid points & $\begin{array}{l}\text { Grid } \\
\text { spac- } \\
\text { ing } h\end{array}$ \\
\hline 1 & $481 \times 161$ & 1 \\
2 & $241 \times 81$ & 2 \\
3 & $121 \times 41$ & 4 \\
\hline
\end{tabular}

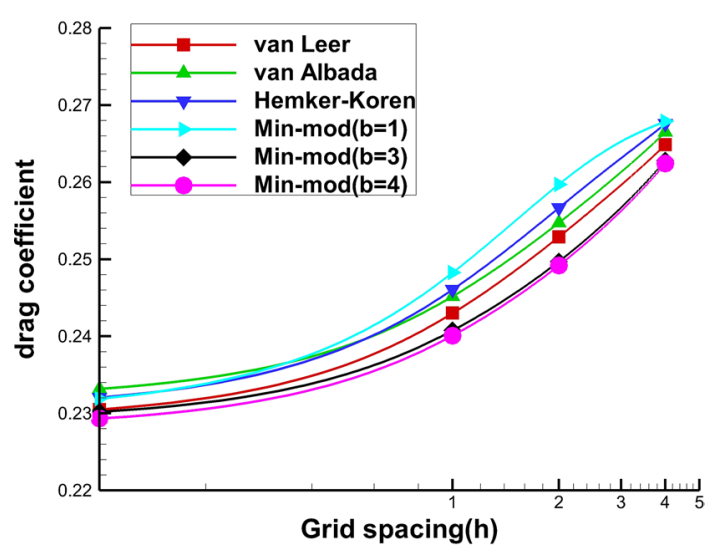

Fig. 17 Comparison of drag coefficients by different limiter functions for compression ramp plate

Figure 18 presents the estimated spatial errors based on the hybrid-order analysis method for all the concerned limiters. The non-monotonic grid-convergence features are observed for all limiter functions. It is clearly showing that, the spatial errors gradually approach to the first-order range on the densest grid, while to the second-order range on the coarsest grid. The sum of them based on the hybrid-order analysis method is also shown in Fig. 19. It can also be obviously seen that Min-mod limiter of $\beta=3,4$ predicts the smallest error, while the highest error with $\beta=1 \mathrm{Min}-\bmod$ limiter, thus the dissipation level of Min-mod-type limiter is determined by the compression parameter: $\beta=3,4$ indicates a compressive limiter with higher accuracy, while $\beta=1$ indicates a dissipative limiter with less accuracy.

The computational results, in terms of the pressure coefficient, skin friction coefficient and heat transfer rate, on the densest grid from different limiter functions are plotted in Fig. 20a-c. The heat transfer rate is defined as $\mathrm{Ch}=k(\partial T / \partial n)_{w} /\left[\rho_{\infty} u_{\infty}\left(H_{\infty}-H_{w}\right)\right], H$ is the total enthalpy with the subscript $w$ standing for the solid surface. As noted by Dolling [1], Knight [2, 4], Zheltovodovo [3, 5] and Rudy [46], the flow field of hypersonic SWBLI over a ramp with large ramp angle is dominated greatly by the unsteady shockwave system and large flow recirculation. From Fig. 20, the wavy distributions of pressure, skin friction and heat transfer along the aft part surface of the compressive ramp are obvious. Because the simulation is performed based on the framework of steady RANS, this wavy features are mainly caused by the low-level numerical dissipation that is not enough to suppress the numerical oscillations in the current strong SWBLI flows, even though the shockwave system in the flowfield, as well as the flow separation, is unsteady in physics.

The distribution of pressure coefficients on the ramp plate is shown in Fig. 20a. The start points of the pressure rise for Min-mod limiters of $\beta=3,4$ are at the location close to $X / L=0.5$, while others at about $X / L=0.6 \sim 0.7$. The peak values of pressure and their locations are markedly different for all limiters. Min-mod limiters of $\beta=3,4$ predict the closest pressure peak value as compared to the experiment data. The peak location of Min-mod limiter of $\beta=4$ is towards the most downstream in the streamwise direction. However, Min-mod limiter of $\beta=1$ obtains the smallest pressure peak value at the most upstream peak location. Therefore, the peak value decreases and peak location moves upstream as the dissipation of limiter functions, as shown in Fig. 20a.

For the skin friction coefficients shown in Fig. 20b, the position of the skin friction turning negative indicates the beginning of the separation bubble, while the position where the skin friction turning back to positive corresponds to the reattachment of the separation bubble. Therefore, Min-mod limiter of $\beta=4$ shows the largest region of negative skin friction, which indicates the largest separation region as compared to that of all other limiters, while Min-mod limiter of $\beta=1$ predicts the smallest separation region. The predicted peak values and their locations of the skin friction on ramp plate vary significantly. It is clear that Min-mod limiters with $\beta=3,4$ predict the similar peak values and locations. However, the peak value of $\beta=1$ Min-mod limiter is the smallest and the location is the most upstream. That clearly implies that the compressive feature would make the predicted peak value larger and the peak location more downstream. From the distribution of heat transfer rate shown in Fig. 20c, it can be seen obviously that the predicted peak values 


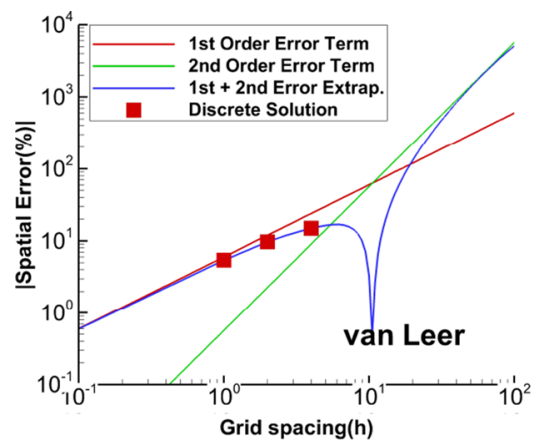

(a)

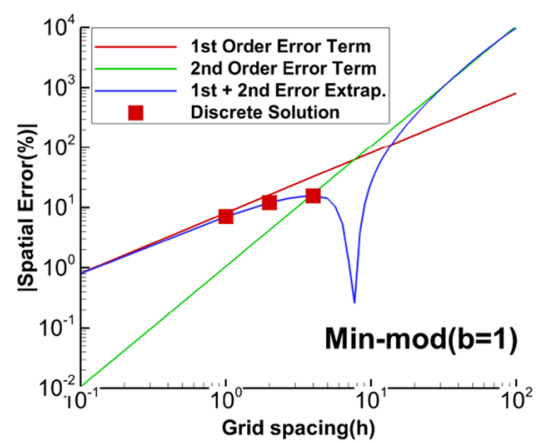

(d)

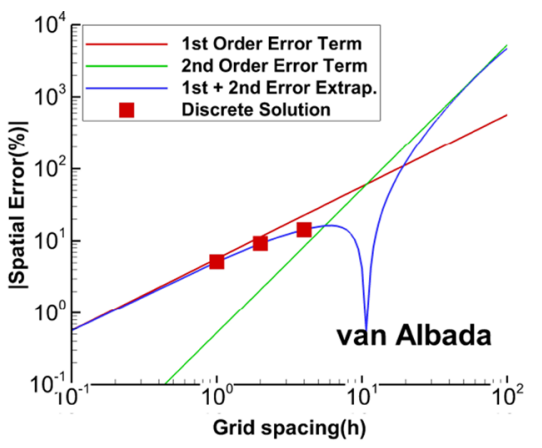

(b)

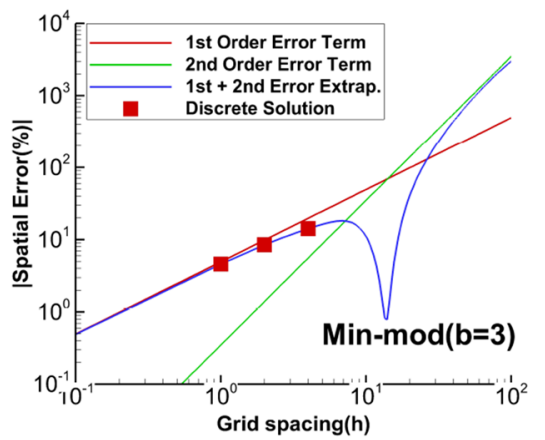

(e)

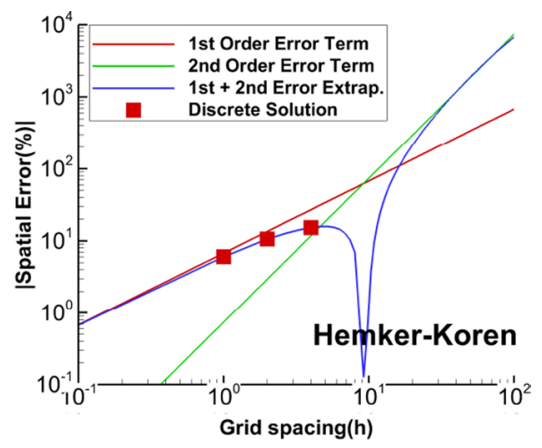

(c)

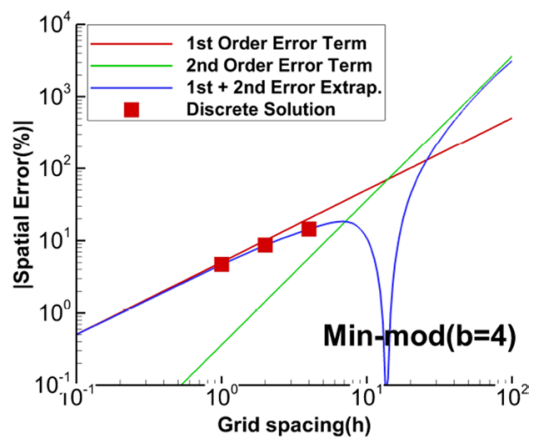

(f)

Fig. 18 Spatial errors for different limiter functions with grid refinement

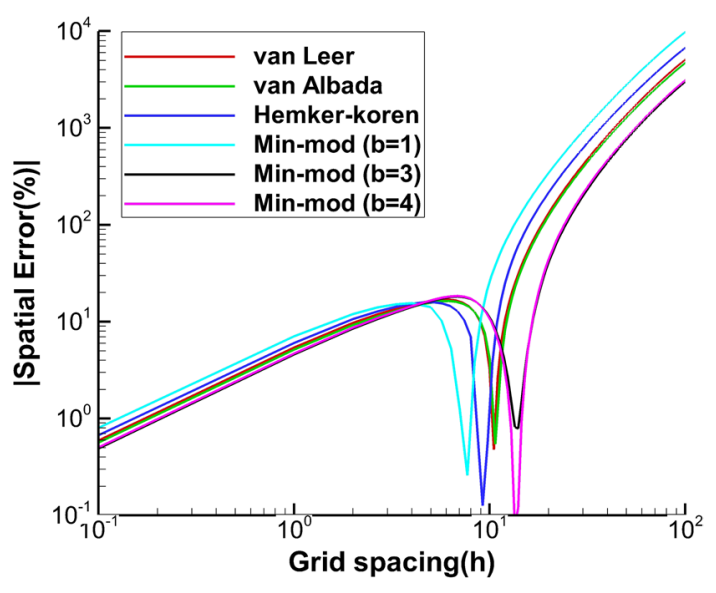

Fig. 19 Comparison of hybrid-order spatial errors for SWBLI on compression ramp plate

and locations exhibit the similar trend with that of skin friction, being distributed in a very scattered manner for different limiters.

Table 6 presents the detail information of the separation region predicted with different limiters, and Min-mod limiter of $\beta=4$ predicts the largest separation region with the most upstream separation point and the most downstream reattachment point. However, the well-known Min-mod limiter of $\beta=1$ captures the smallest separation region with the most downstream separation point and the most upstream reattachment point. The size of the flow separation regions by different limiters varies greatly. The more compressive the limiter function, the larger the predicted separation region size. This is consistent with the results of surface skin friction distribution shown in Fig. 20b, which indicates the similar conclusion by the range of negative skin friction values. Therefore, Min-mod-type limiter can be compressive with the reasonable compression parameter, not always be dissipative as mentioned in most of the previous literature.

A qualitative investigation about the concerned SWBLI flow features is performed according to the contours of velocity divergence shown in Fig. 21. It is obvious that the flow features by different limiters show great discrepancy because of their different dissipation levels. With a compressive limiter, such as Min-mod limiter of $\beta=4$, the predicted separated flow region on the ramp plate is larger. The compression waves caused by the separation bubble firstly combines with the shockwave developed from the leading edge of the horizontal plate. Then the merged shock wave interacts with the expansion fans and re-compression waves. However, with a dissipative limiter such as Min-mod limiter of $\beta=1$, the compression waves, expansion fans and re-compression waves around the separation bubble firstly coalesce into a shockwave, and then the coalesced shockwave interacts with the leading edge shockwave. The different manners of shockwaves interacting with boundary layer 


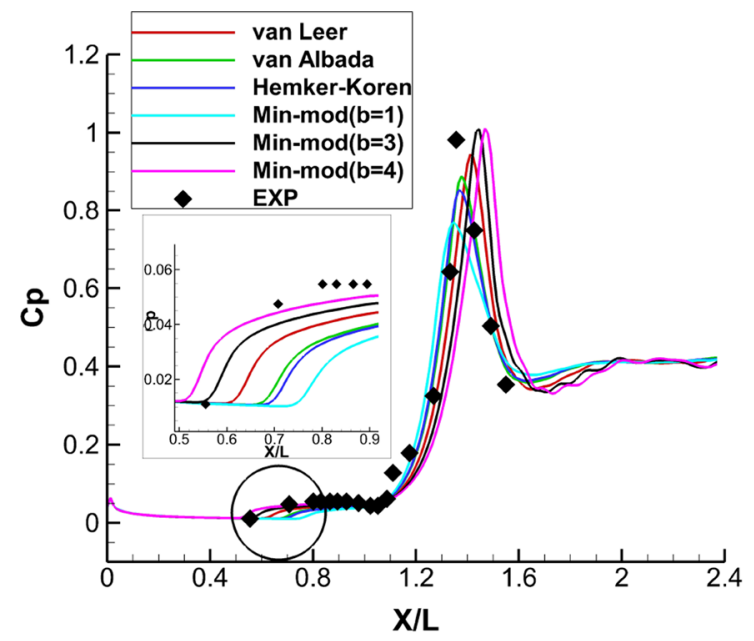

(a) Pressure coefficient

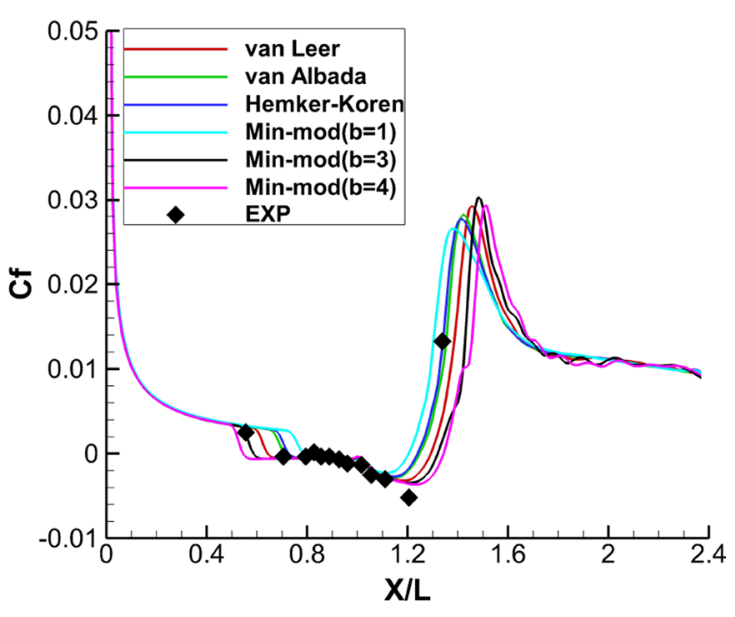

(b) Skin friction coefficient

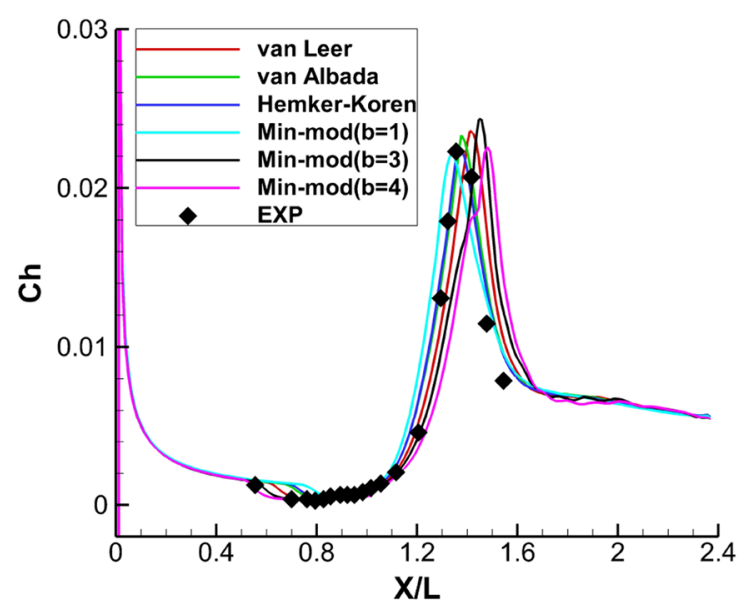

(c) Non-dimensional heat transfer rate

Fig. 20 Computational results of the compression ramp plate. a Pressure coefficient, $\mathbf{b}$ skin friction coefficient, $\mathbf{c}$ non-dimensional heat transfer rate

Table 6 Detailed information about separation region with different limiters

\begin{tabular}{llll}
\hline Limiter function & $\begin{array}{l}\text { Separation } \\
\text { point }\left(X_{\mathrm{S}} / L\right)\end{array}$ & $\begin{array}{l}\text { Reattachment } \\
\text { point }\left(X_{\mathrm{R}} / L\right)\end{array}$ & Separation length \\
\hline van Leer & 0.643465 & 1.29232 & 0.648855 \\
van Albada & 0.71119 & 1.24822 & 0.537030 \\
Hemker-Koren & 0.727391 & 1.23974 & 0.512349 \\
Min-mod $\beta=1$ & 0.786031 & 1.20417 & 0.418139 \\
Min-mod $\beta=3$ & 0.586151 & 1.31993 & 0.733779 \\
Min-mod $\beta=4$ & 0.545169 & 1.34315 & 0.797981 \\
\hline
\end{tabular}

result in the variations of computational results regarding the peak value and location for pressure, skin friction and heat transfer rate. The flow structure predicted by the most dissipative Min-mod limiter of $\beta=1$ is obviously different with that of Min-mod limiter of $\beta=3$, 4 . In addition, the wavy distributions after the shockwave interaction point observed in the contours of Min-mod limiter of $\beta=3,4$ are caused by their overly compressive properties.

Based on the above discussions about these two strong shockwave-containing SWBLI problems in Sects. 5.2 and 5.3 , the numerical solution accuracy is greatly influenced by both the intrinsic dissipation level and the iterative property of the employed limiter. However, the intrinsic dissipation dominates over its iterative property relating to the differentiable/non-differentiable features, especially in the flow field that contains strong discontinuous phenomena. Although the residuals of Min-mod limiter of $\beta=3,4$ do not converge to the machine zero, they can still predict the solution with the higher accuracy than that of the commonly 


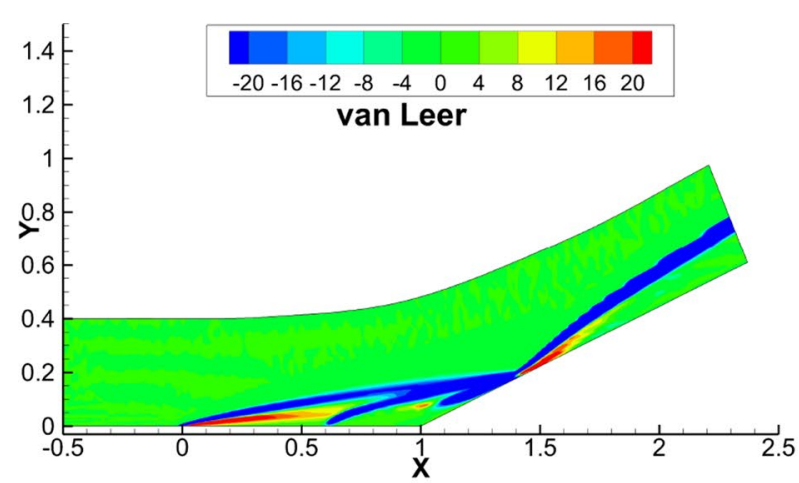

(a)

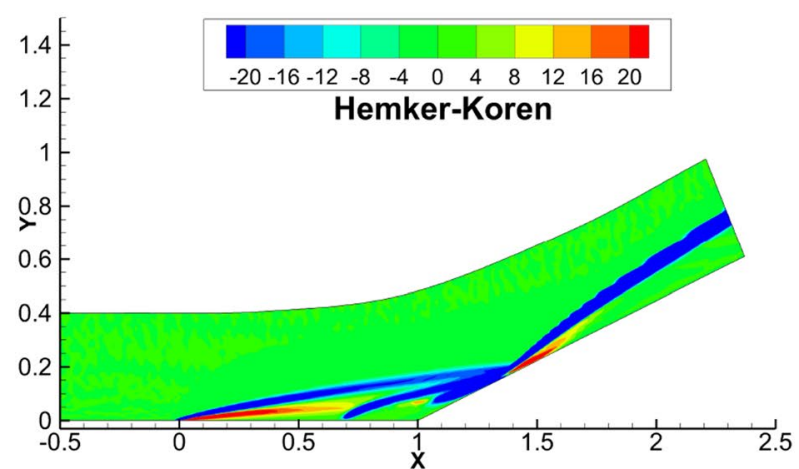

(c)

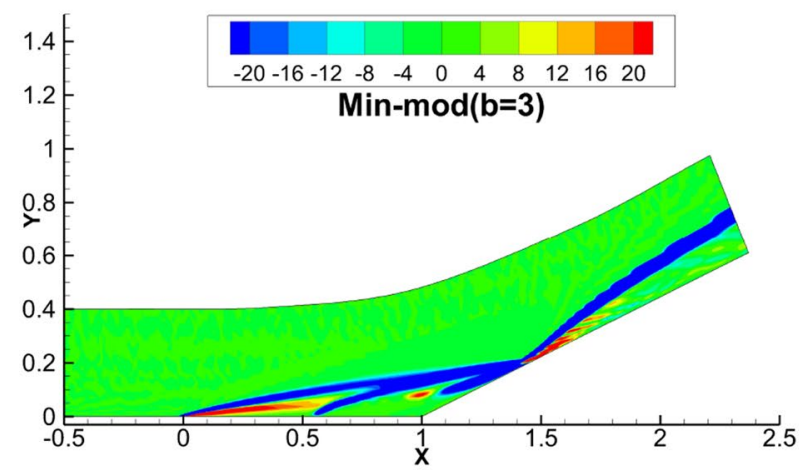

(e)

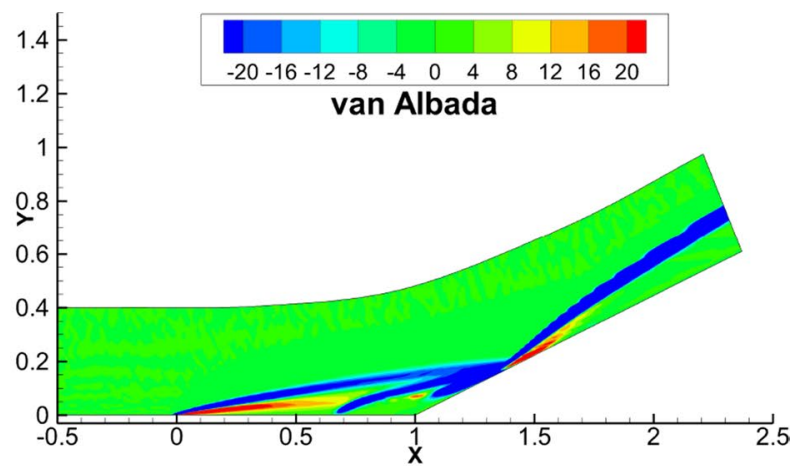

(b)

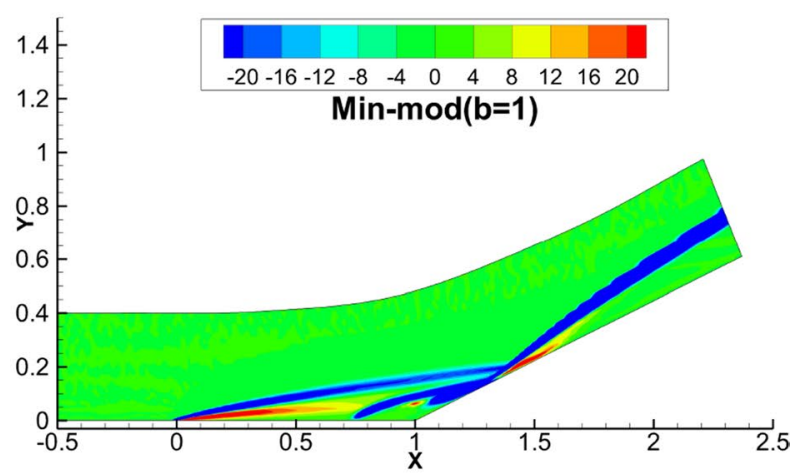

(d)

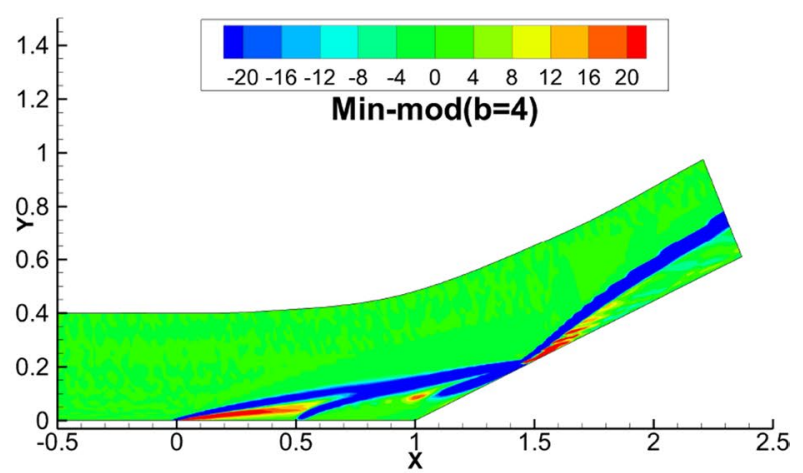

(f)

Fig. 21 Contours of velocity divergence with different limiters over the compression ramp plate

used limiter functions as shown in Fig. 2. Thus, Min-mod limiter definitely is a compressive limiter with $\beta=3,4$, but be a dissipative limiter with $\beta=1$, which implies Min-mod limiter can also achieve high-order accuracy for shockwavecontaining flows with reasonable compression parameter $\beta$.

\section{Conclusions}

The compressive properties of Min-mod-type limiter are investigated theoretically and numerically in the current research. As a comparison, a series of commonly used MUSCL-based limiter functions are assessed together.
Three typical shockwave-containing flows, including the transonic flow about NACA 0012 airfoil and two highspeed laminar SWBLI problems, are performed. A hybridorder spatial error estimator is introduced for the first time in public literature to perform the limiter function investigations. Some key conclusions can be drawn as follows:

1. The MUSCL-based interpolation commonly used to obtain formally second-order accurate scheme is reformulated according to Sweby's second-order TVD limiter region and Spekreijse's second-order monotonic limiter region. The properties of Min-mod-type limiter are found to be determined by the compression parameter 
$\beta$. This notion complements the previous incomplete comments on Min-mod-type limiter by demonstrating its compressive properties, not only the dissipative properties.

2. The hybrid-order spatial error estimator devised by Roy is introduced for the first time to perform the analysis and comparison of limiter functions' dissipative characteristics. Studies have shown that the inherent dissipation of the limiter function has a significant impact on the numerical solution accuracy in shockwave-containing flows. The compressive limiter, like Min-mod limiter of $\beta=4$, can predict the flow discontinuities more accurately than a dissipative limiter does.

3. It is very hard to obtain the well-converged solution in strong shockwave-containing flows, such as the selected supersonic/hypersonic SWBLI problems, no matter the limiter is differentiable or non-differentiable, and the differentiable/non-differentiable properties of limiters are no longer the indication of the iterative convergence to machine zero for strong shockwave-containing flows.

4. This work demonstrates that not only theoretically but also numerically Min-mod-type limiter changes from the dissipative limiter to the compressive limiter by selecting the reasonable compression parameter. The compressive properties of Min-mod-type limiter have been substantiated in current research through simulating strong shockwave-containing flows, resulting in high resolution of flow structures. This is significant for the further development of new numerical schemes via Minmod-like procedure to simulate strong discontinuitycontaining problems more accurately.

Open Access This article is licensed under a Creative Commons Attribution 4.0 International License, which permits use, sharing, adaptation, distribution and reproduction in any medium or format, as long as you give appropriate credit to the original author(s) and the source, provide a link to the Creative Commons licence, and indicate if changes were made. The images or other third party material in this article are included in the article's Creative Commons licence, unless indicated otherwise in a credit line to the material. If material is not included in the article's Creative Commons licence and your intended use is not permitted by statutory regulation or exceeds the permitted use, you will need to obtain permission directly from the copyright holder. To view a copy of this licence, visit http://creativecommons.org/licenses/by/4.0/.

\section{References}

1. Dolling DS (2012) Fifty years of shock-wave/boundary-layer interaction research-what next? AIAA J 39:1517-1531. https:// doi.org/10.2514/3.14896

2. Knight D, Yan H, Panaras A, Zheltovodov A (2013) RTO WG 10 - CFD validation for shock wave turbulent boundary layer interactions. AIAA Pap. https://doi.org/10.2514/6.2002-437
3. Zheltovodov A (2012) Some advances in research of shock wave turbulent boundary layer interactions. In: 44th AIAA aerospace sciences meeting and exhibit 9-12 January 2006, Reno, Nevada. American Institute of Aeronautics and Astronautics, pp 1-25

4. Knight D, Yan H, Panaras AG, Zheltovodov A (2003) Advances in CFD prediction of shock wave turbulent boundary layer interactions. Prog Aerosp Sci 39:121-184. https://doi.org/10.1016/ S0376-0421(02)00069-6

5. Zheltovodov AA (2004) Advances and problems in modeling of shock wave turbulent boundary layer interactions. In: Proceedings of the international conference on the methods of aerophysical research, Novosibirsk, Russia, Part I. pp 225-236

6. Xin-Liang L, De-Xun F, Yan-Wen M (2006) Direct numerical simulation of a spatially evolving supersonic turbulent boundary layer at $\mathrm{Ma}=6$. Chin Phys Lett 23:1519-1522. https://doi. org/10.1088/0256-307x/23/6/045

7. Zhao H, Balakumar P (2005) Nonlinear disturbance evolution across a hypersonic compression corner. AIAA J 43:1034-1041. https://doi.org/10.2514/1.2244

8. Yee HC, Sandham ND, Djomehri MJ (1999) Low-dissipative high-order shock-capturing methods using characteristic-based filters. J Comput Phys 150:199-238. https://doi.org/10.1006/ jcph.1998.6177

9. Wang ZJ (2007) High-order methods for the Euler and NavierStokes equations on unstructured grids. Prog Aerosp Sci 43:141. https://doi.org/10.1016/j.paerosci.2007.05.001

10. Shu C (2016) High-order finite difference and finite volume WENO schemes and discontinuous Galerkin methods for CFD high-order finite difference and finite volume WENO schemes and discontinuous Galerkin methods for CFD. Int J Comput Fluid Dyn 8562:106-118. https://doi.org/10.1080/1061856031 000104851

11. Ekaterinaris JA (2005) High-order accurate, low numerical diffusion methods for aerodynamics. Prog Aerosp Sci 41:192-300. https://doi.org/10.1016/j.paerosci.2005.03.003

12. Venkatakrishnan V, Allmaras S, Kamenetskii D, Johnson F (2012) Higher order schemes for the compressible Navier-Stokes equations. AIAA Pap. https://doi.org/10.2514/6.2003-3987

13. Steger JL, Warming R (1981) Flux vector splitting of the inviscid gas-dynamic equations with applications to finite difference methods. J Comput Phys 40:263-293. https://doi.org/10.1016/00219991(81)90210-2

14. Müller E (1988) Flux-vector splitting for the Euler equations for real gases. J Comput Phys 79:227-230. https://doi. org/10.1016/0021-9991(88)90013-7

15. Roe PL (1997) Approximate Riemann solvers, parameter vectors, and difference schemes. J Comput Phys 135:250-258. https://doi. org/10.1006/jcph.1997.5705

16. Knight DD, Degrez G (1998) Shock wave boundary layer interactions in high mach number flows. NATO Res Technol Organ $2: 1-35$

17. Harten A (2005) On a class of high resolution total-variationstable finite-difference schemes. SIAM J Numer Anal 21:1-23. https://doi.org/10.1137/0721001

18. Osher S, Chakravarthy S (2005) High resolution schemes and the entropy condition. SIAM J Numer Anal 21:955-984. https://doi. org/10.1137/0721060

19. Sweby PK (1985) High resolution TVD schemes using flux limiters. Lect Appl Math 22:289-309

20. Yee HC (1987) Upwind and symmetric shock- capturing schemes. NASA Tech Memo 89464:130

21. Van Leer B (1979) Towards the ultimate conservation difference scheme V: a second-order sequel to Godunov's method. J Comput Phys 136:101-136. https://doi.org/10.1016/0021-9991(79)90145 $-1$ 
22. Engquist B, Osher S (1981) One-sided difference approximations for nonlinear conservation laws. Math Comput 36:321-352. https ://doi.org/10.2307/2007646

23. Sweby PK (1984) High resolution schemes using flux limiters for hyperbolic conservation laws. SIAM J Numer Anal 21:995-1011. https://doi.org/10.1137/0721062

24. Goodman JB, LeVeque RJ (1985) On the accuracy of stable schemes for 2D scalar conservation laws. Math Comput 45:15-21. https://doi.org/10.1090/S0025-5718-1985-0790641-4

25. Spekreijse $S$ (1987) Multigrid solution of monotone second-order discretizations of hyperbolic conservation laws. Math Comput 49:135-155. https://doi.org/10.2307/2008254

26. Barth T, Jespersen D (1989) The design and application of upwind schemes on unstructured meshes. In: 27th aerospace science meeting AIAA-89-03: https://doi.org/10.2514/6.1989-366

27. Venkatakrishnan V (1995) Convergence to steady state solutions of the Euler equations on unstructured grids with limiters. J Comput Phys 118:120-130

28. van Albada GD, van Leer B, Roberts WW (2011) A comparative study of computational methods in cosmic gas dynamics. Upwind High-Resolut Schemes 108:95-103. https://doi.org/10.1007/9783-642-60543-7_6

29. Kim KH, Kim C (2005) Accurate, efficient and monotonic numerical methods for multi-dimensional compressible flows. J Comput Phys 208:570-615. https://doi.org/10.1016/j.jcp.2005.02.022

30. Yoon SH, Kim C, Kim KH (2009) Multi-dimensional limiting process for two-and three-dimensional flow physics analyses. In: Computational fluid dynamics 2006-Proceedings of the fourth international conference on computational fluid dynamics, ICCFD, vol 227, pp 185-190. https://doi.org/10.1007/978-3-54092779-2-27

31. Scott J, Niu Y (1993) Comparison of limiters in flux-split algorithms for Euler equations. AIAA Pap. https://doi. org/10.2514/6.1993-68

32. Li G, Bhatia D, Xu M, Wang J (2019) Grid convergence analysis for muscl-based numerical scheme in shockwave-containing flows. MATEC Web Conf 257:02001. https://doi.org/10.1051/ matecconf/201925702001

33. Chao Y, Liang C (1996) The scheme effects of numerical solutions for shock/boundary layer interaction. ACTA Aeronaut Astronaut Sin 17:67-70

34. Hemker PW, Koren B (1988) Multigrid, defect correction and upwind schemes for the steady Navier-Stokes equations. CWI. Department of Numerical Mathematics
35. Li G, Zhou Z (2010) Validation of a multigrid-based NavierStokes solver for transonic flows. AIAA Pap. https://doi. org/10.2514/6.2010-4549

36. Kim KH, Kim C, Rho OH (2001) Methods for the accurate computations of hypersonic flows: I. AUSMPW + scheme. J Comput Phys 174:38-80

37. Liou M-S, Steffen CJ (1991) A new Flux splitting scheme. J Comput Phys 107:23-39. https://doi.org/10.1006/jcph.1993.1122

38. Anderson WK, Thomas JL, Van Leer B (1986) Comparison of finite volume flux vector splittings for the Euler equations. AIAA J 24:1453-1460

39. Roache PJ (1998) Verification and validation in computational science and engineering, vol 895. Hermosa, Albuquerque, NM

40. Roache PJ (1994) Perspective: a method for uniform reporting of grid refinement studies. J Fluids Eng 116:405. https://doi. org/10.1115/1.2910291

41. Roy CJ (2003) Grid convergence error analysis for mixed-order numerical schemes. AIAA J 41:595-604

42. Roy CJ, McWherter-Payne MA, Oberkampf WL (2008) Verification and validation for laminar hypersonic flowfields, part 1: verification. AIAA J 41:1934-1943. https://doi.org/10.2514/2.1909

43. Roy CJ, McWherter-Payne MA, Oberkampf WL (2000) Verification and validation for laminar hypersonic flowfields. AIAA Pap. https://doi.org/10.2514/6.2000-2550

44. Hakkinen RJ, Greber I, Trilling L, Abarbanel SS (1959) The interaction of an oblique shock wave with a laminar boundary layer. NASA Memo 2-18-59W

45. Holden MS, Moselle JR (1970) Theoretical and experimental studies of the shock wave-boundary layer interaction on compression surfaces in hypersonic flow. ARL 70-0002, Aerospace Research Laboratories, Wright-Patterson AFB, OH. https://doi. org/10.21236/ad0706135

46. Rudy DH, Thomas JL, Kumar A et al (1991) Computation of laminar hypersonic compression-corner flows. AIAA J 29(7):11081113. https://doi.org/10.2514/3.10710

Publisher's Note Springer Nature remains neutral with regard to jurisdictional claims in published maps and institutional affiliations. 